\title{
Two New Series of Principles in the Interpretability Logic of all Reasonable Arithmetical Theories
}

\author{
Evan Goris, Joost J. Joosten*
}

May 20, 2020

\begin{abstract}
The provability logic of a theory $T$ captures the structural behavior of formalized provability in $T$ as provable in $T$ itself. Like provability, one can formalize the notion of relative interpretability giving rise to interpretability logics. Where provability logics are the same for all moderately sound theories of some minimal strength, interpretability logics do show variations.

The logic $\mathbf{I L}($ All) is defined as the collection of modal principles that are provable in any moderately sound theory of some minimal strength. In this paper we raise the previously known lower bound of IL(All) by exhibiting two series of principles which are shown to be provable in any such theory. Moreover, we compute the collection of frame conditions for both series.
\end{abstract}

\section{Introduction}

Relative interpretations in the sense of Tarski, Mostowski and Robinson [12] are widely used in mathematics and in mathematical logic to interpret one theory into another. Roughly speaking, such an interpretation between two theories is a translation from the language of one theory to the language of the other so that the translation preserves logical structure and theoremhood.

We shall write $U \triangleright V$ to denote that a theory $U$ interprets a theory $V$. Once we know that $U \triangleright V$, this provides us much information; for example the consistency of $U$ implies the consistency of $V$ and also, various definability results

\footnotetext{
${ }^{*}$ Keywords: Interpretability logics, interpretations, definable cuts, weak arithmetics, formal provability (03B45, 03B60, 03F 30, 03F40, 03F 45); The second author has received support from the Generalitat of Catalunya under Grant 2017 SGR 270, from the Secretary of Universities and Research of the Department of Economy and Knowledge of the Generalitat of Catalonia (Grants 2016 DI 032 and 2016 DI 033), from the Spanish Ministry of Economy and Competitiveness under grant Number FFI2015-70707P and from the Spanish Ministry of Science and Universities under grant Number RTC-2017-6740-7.
} 
carry over from the one theory to the other. Famous examples of interpretations are abundant: the theory of the natural numbers into the theory of the integers, set theory plus the continuum hypothesis into ordinary set theory (in fact, set theory plus the negation of the continuum hypothesis can also be interpreted into ordinary set theory, though this fact is less well-known), non-Euclidean geometry into Euclidean geometry, etc.

Interpretability, being a syntactical notion, allows for formalization very much as one can formalize the notion of provability. As such, we can consider interpretability logics which will actually extend the well-know provability logic GL named after Gödel and Löb. We shall see that the interpretability logic of a theory is the collection of all structural properties of interpretability that it can prove.

Where all modestly correct theories of some minimal strength — let us call them reasonable theories in this paper- have the same provability logic GL, the situation is different in the case of interpretability and different theories have different logics. It is an open question to determine the logic of interpretability principles being provable in any reasonable theory. This paper reports on substantial progress on this open question by increasing the previously known lower bound.

\section{Preliminaries}

Let $U$ and $V$ denote theories with languages $\mathcal{L}_{U}$ and $\mathcal{L}_{V}$ respectively. A relative interpretation $j$ from $V$ into a theory $U$-we will write $j: U \triangleright V$ - is a pair $\langle\delta(x), t\rangle$ where $\delta(x)$ is a formula of $\mathcal{L}_{U}$ that specifies the domain in which $V$ will be interpreted and $t$ is a translation, mapping symbols of $\mathcal{L}_{V}$ to formulas of $\mathcal{L}_{U}$ providing a definition in $U$ of these symbols.

For further details, we refer the reader to [16] and just mention some particularities here. For example, we restrict to languages with only constant and relations symbols and treat function symbols as functional relations. Moreover, we restrict ourselves to one-dimensional interpretations where each object in the one theory is represented as one object in the other theory, as opposed to a sequence of objects, as in the case of higher dimensional interpretations as studied, for example in [10].

Further, we do not allow for extra parameters in our interpretation. Thus, an $n$-ary relation symbol $R$ in the language of $V$ will be mapped by $t$ to a formula $R^{t}$ in the language of $U$ with exactly $n$ free variables. If the language of $V$ contains equality, we do not require that $t$ maps equality to equality.

The translation $t$ is extended to a translation $j$ of formulas in the usual way by having $j$ commute with the connectives and relativize the quantifiers to the domain specifier $\delta(x)$ as follows: $(\forall x \varphi(x))^{j}:=\forall x\left(\delta(x) \rightarrow \varphi^{j}(x)\right)$. We will not go too much into details but the main point is that interpretations are primarily syntactical notions - especially for finite languages - and as such allow for an arithmetization/formalization very much as formal proofs do. 


\subsection{Arithmetic}

In order to formalize the notion of interpretability within some base theory $T$ one needs to require some minimal strength conditions on $T$. In particular, we shall require that $T$ can speak of numbers where to code syntax and we shall assum 1 that the language of $T$ contains the language of arithmetic $\langle+, \times, S, 0,1<,=\rangle$.

We will need that the main properties of the basic syntactical operations like substitution are provable within $T$. For reasonable coding protocols this implies that we need to require the totality of a function of growth-rate $\omega_{1}(x):=x \mapsto$ $2^{|x|^{2}}$ where $|x|$ denotes the integral part of the binary logarithm of $x$.

Further, to perform basic arguments we need a minimal amount of induction and actually a surprisingly little amount of induction suffices. Buss's theory $\mathrm{S}_{2}^{1}$ has just the needed amount of induction and proves the totality of $\omega_{1}$ and this shall be our base theory (formulated in the standard language of arithmetic).

Alternatively, we could have taken as base theory I $\Delta_{0}+\Omega_{1}$ which consists of Robinson's arithmetic $Q$ together with induction for bounded formulas with parameters and the axiom $\Omega_{1}$ stating that the graph of $\omega_{1}$ defines a total function. We refer the reader to [5] and [2] for further details.

A sharply bounded quantifier is one of the form $\forall x<|y|$ where $|y|$ denotes the integer value of the binary logarithm of $y$. The class $\Delta_{0}^{b}$ contains exactly the formulas where each quantifier is sharply bounded. The class $\Sigma_{1}^{b}$ arises by allowing bounded existential quantifiers and sharply bounded universal quantifiers to occur over $\Delta_{0}^{b}$ formulas. It is essential that the $\omega_{1}$ function may occur in the bounding terms. By $\exists \Sigma_{1}^{b}$ we denote those formulas that arise by allowing a single unbounded existential quantifier over a $\Sigma_{1}^{b}$ formula. The complexity classes $\Pi_{n}, \Sigma_{n}$ and $\Delta_{n}$ refer to the usual quantifier alternations hierarchies in the standard language of arithmetic.

In this paper we shall only be concerned with first order-theories containing the language of arithmetic with a poly-time recognizable set of axioms extending $S_{2}^{1}$ and shall often refrain from repeating (some of) these conditions. We shall write $\square_{T} \phi$ as the $\exists \Sigma_{1}^{b}$ formalization of $\phi$ being provable in the theory $T$ and refrain from distinguishing formulas from their Gödel numbers or even the numerals thereof. When $I$ is a formula with one free variable we shall denote by $\square_{T}^{I} \phi$ the formalization of $\phi$ being provable in the theory $T$ with a proof that satisfies $I$. It is well known that we can express provable $\Sigma_{1}$ completeness using formalized provability.

Lemma 2.1. For any theory $T$ extending $\mathrm{S}_{2}^{1}$ we have that

$$
T \vdash \forall \alpha\left(\square_{T} \alpha \rightarrow \square_{T} \square_{T} \alpha\right) .
$$

\footnotetext{
${ }^{1}$ One can consider a slightly more general setting where theories $T$ do not directly speak of the natural numbers but where it is assumed that $T$ is decent in some sense and comes with an interpretation $\mathcal{N}$ of the natural numbers (see e.g. [17]). One then only has to require that $\mathcal{N}$ satisfies sufficiently many axioms of number theory so that the arithmetisation of syntax can be performed. For example, ZFC set theory does not contain the language of arithmetic but we can easily perceive the numbers as 'living' inside set theory, that is, there is a natural interpretation of the numbers in ZFC.
} 
We will use $U \triangleright V$ to denote the formalization of "the theory $V$ is interpretable in the theory $U$ ". If we abbreviate the existential quantifier over numbers that code a pair $\langle\delta(x), t\rangle$ defining an interpretation by $\exists^{\text {int }} j$ we can write

$$
U \triangleright V:=\exists^{\text {int }} j \forall \psi\left(\square_{V} \psi \rightarrow \square_{U} \psi^{j}\right) .
$$

An interpretation $j: U \triangleright V$ can be used as a uniform way to obtain a model of $V$ inside any model of $U$. If $U$ satisfies full induction, then we see that actually the defined model of $V$ is an end extension of the model of $U$ : we define $f(0):=0$ and $f(x+1):=f(x)+{ }^{j} 1^{j}$ and by induction see that $\forall x \exists y f(x)=y$. As such, we see that any $\Sigma_{1}$ consequence of $U$ must necessarily also hold in $V$. Since $\square_{T} \varphi$ is a $\Sigma_{1}$ formula, the insight on end extensions is reflected in what is called Montagna's principle

$$
(U \triangleright V) \rightarrow\left(\left(U \cup\left\{\square_{T} \varphi\right\}\right) \triangleright\left(V \cup\left\{\square_{T} \varphi\right\}\right)\right) .
$$

In case $U$ does not have full induction, we can still define the graph $F(x, y)$ of the function $f$ from above, but we can no longer prove that the function is total. However, we can prove that $\exists y F(x, y)$ is progressive, that is, we can prove

$$
\exists y F(0, y) \wedge \forall x(\exists y F(x, y) \rightarrow \exists y F(x+1, y)) .
$$

In particular, the formula $\forall x^{\prime} \leq x \exists y F\left(x^{\prime}, y\right)$ defines an initial segment within $U$. A common trick in weak arithmetics is to use this initial segment as our natural numbers instead of applying induction (which is not necessarily available). By Solovay's techniques on shortening initial segments we may assume that they obey certain closure properties giving rise to the what is called a definable cut.

A formula $J$ is called a $T$-cut whenever $T$ proves all of

1. $J(0) \wedge \forall x(J(x) \rightarrow J(x+1))$;

2. $J(x) \wedge y<x \rightarrow J(y)$;

3. $\forall x \forall y\left(J(x) \wedge J(y) \rightarrow J(x+y) \wedge J(x y) \wedge J\left(\omega_{1}(x)\right)\right)$.

Let $\operatorname{Cut}(J)$ denote the conjunction of these three requirements. Sometimes we want to quantify over cuts within $T$ so that these cuts can then of course be non-standard. We shall use $\forall^{\text {Cut }} J \psi$ and $\exists^{\text {Cut }} J \psi$ to denote $\forall J\left(\square_{T}\right.$ Cut $(J) \rightarrow \psi$ ) and $\exists J\left(\square_{T} \operatorname{Cut}(J) \wedge \psi\right)$ respectively. Sometimes we shall write $x \in J$ instead of $J(x)$.

Sometimes we will need to find a cut $J$ inside another cut $I$. In such cases we will not just require that the Gödel number of the formula $J$ is in $I$ but moreover we shall require that the proof that $J$ is a cut can also be found within $I$. Thus, for example, $\exists^{\text {Cut }} J \in I \psi$ will be short for $\exists J\left(\square_{T}^{I}\right.$ Cut $\left.(J) \wedge \psi\right)$.

We note that if $\psi(v) \in \exists \Sigma_{1}^{b}$, then $\exists^{\text {Cut }} J \psi(J)$ is again provably equivalent to an $\exists \Sigma_{1}^{b}$ formula.

Let us get back to the role of induction in Montagna's principle. If $j: U \triangleright V$ and $U$ does not prove full induction, then $j$ will not define an end extension 
of any model of $U$. However, it is easy to see that $j$ does define, using the progressive formula $\forall x^{\prime} \leq x \exists y F\left(x^{\prime}, y\right)$, a definable cut in $U$ on which $f$ is an isomorphism. This is reflected in a weakening of Montagna's principle also referred to as Pudlák's principle.

Lemma 2.2. Let $T$ be a theory containing $\mathrm{S}_{2}^{1}$ and let $U$ and $V$ be theories.

$$
T \vdash U \triangleright V \rightarrow \exists^{\text {Cut }} J \forall \psi \in \Delta_{0}\left(U \cup\left\{(\exists x \psi)^{J}\right\} \triangleright V \cup\{\exists x \psi\}\right) .
$$

\subsection{The interpretability logic of a theory}

Interpretability logics are designed to capture structural behavior of formalized interpretability just as provability logic captures the structural behavior of formalized provability. To this end we consider a propositional modal language with a unary modal operator $\square$ to model formalized provability and a binary modal operator $\triangleright$ to model formalized interpretability of sentential extensions of some base theory. Let us make this more precise.

Thus, let us fix an arithmetical theory $T$; By $*$ we will denote a realization, that is, any mapping from the set of propositional variables to sentences of $T$. The map $*$ is extended to the set of all modal formulas of interpretability logics as follows

$$
\begin{array}{lll}
(\perp)^{*} & :=0=1 & \\
(\neg A)^{*} & :=\neg A^{*} & \\
(A \wedge B)^{*} & :=A^{*} \wedge B^{*} & \text { and likewise for the other connectives } \\
(\square A)^{*} & :=\square_{T} A^{*} & \\
(A \triangleright B)^{*} & :=\left(T+A^{*}\right) \triangleright\left(T+B^{*}\right) . &
\end{array}
$$

Note that we use the same symbol $\triangleright$ for the binary modal operator as for the sentence in the language of arithmetic as defined in (1). We can now define the interpretability logic of a theory as those modal principles which are provable under any realization. With some liberal notation this is captured in the following.

Definition 2.3. Let $T$ be a theory containing $\mathrm{S}_{2}^{1}$. We define the interpretability logic of $T$ as

$$
\mathbf{I L}(\mathrm{T}):=\left\{A \mid \forall * T \vdash A^{*}\right\} .
$$

Further, we define the interpretability logic of all arithmetical theories extending $\mathrm{S}_{2}^{1}$ by

$$
\mathbf{I L}(\text { All }):=\left\{A \mid \forall T \forall * T \vdash A^{*}\right\} .
$$

\subsection{Small witnesses}

As a direct corollary to (2) - Montagna's principle - we can conclude that

$$
(A \triangleright B) \rightarrow((A \wedge \square C) \triangleright(B \wedge \square C)) \in \mathbf{I L}(\mathrm{T})
$$


whenever $T$ proves full induction. However, there is no direct reflection of Pudlák's principle on the level of interpretability logics since Pudlák's principle would translate to

$$
(A \triangleright B) \rightarrow\left(\left(A \wedge \square^{J} C\right) \triangleright(B \wedge \square C)\right) \in \mathbf{I L}(\mathrm{T})
$$

for the particular cut $J$ corresponding to $j: A \triangleright B$ and this cannot be expressed in our modal language. In a sense, $\square^{J} C$ corresponds to finding a small witness of the provability of $C$. As we shall see, there are various occasions where we can conclude that such small witnesses exist. The main ingredient in obtaining such small witnesses is expressed by the so-called Outside big, inside small lemma.

To formulate the lemma we should first provide a means to speak under the scope of a provability predicate about numbers that are given externally. As usual this is done via the notion of numerals. A numeral is a syntactical term that uniquely denotes a number. Since unary numerals grow too big, we will resort to dyadic numerals. Dyadic numerals $\tilde{n}$ are defined recursively by $\tilde{0}:=0, \widetilde{2 n}:=S S 0 \times \tilde{n}$ and, $\widetilde{2 n+1}:=S(S S 0 \times \tilde{n})$. Clearly, dyadic numerals are exponentially much shorter than unary numerals.

Lemma 2.4 (Outside big, inside small). For $T, U$ any theories extending $\mathrm{S}_{2}^{1}$, we have that

$$
T \vdash \forall{ }^{\text {Cut }} J \forall x \square_{U} J(\widetilde{x}) .
$$

Proof. Given $J$ and given $x$, not necessarily in $J$, we can construct a proofobject to the extent that $x \in J$ in the obvious way. A proof of $\tilde{n} \in J$ will follow the built-up of $\tilde{n}$ using the standard proofs of lemmas to the effect that $\forall x(x \in J \rightarrow(S S 0 \times x) \in J)$ and $\forall x(x \in J \rightarrow S(S S 0 \times x) \in J)$. We refer to e.g. [2, 7] for details.

The next lemma tells us that small witnesses suit the purpose of inner model constructions.

Lemma 2.5 (Formalized Henkin construction). For theories $T, U$ and $V$ all extending $\mathrm{S}_{2}^{1}$ we have

$$
T \vdash \forall^{\text {Cut }} J\left(U \cup\left\{\operatorname{Con}^{J}(V)\right\} \triangleright V\right) .
$$

Proof. (Sketch) The theory $T$ can verify that the usual Henkin construction can be formalized in $U$ without many problems where $J$ plays the role of the natural numbers. Instead of applying induction to obtain a maximal consistent set $\mathcal{M}_{V}$ as a consistent branch of infinite length in Lindenbaum's lemma, we can now only conclude that the length of the branch is within some cut $I$ which is a shortening of $J$ thereby yielding a set $\mathcal{M}_{V}^{I}$ which is contradiction-free on $I$.

The set $\mathcal{M}_{V}^{I}$ can be used to obtain a term model and we define an interpretation $j:\left(U \cup\left\{\operatorname{Con}^{J}(V)\right\} \triangleright V\right)$ from the term model as usual so that provably $\phi^{j} \leftrightarrow\left(\phi \in \mathcal{M}_{V}^{I}\right)$. Note that since the interpretation of identity can be any equivalence relation, there is no need to move to equivalence classes in the construction of our term model. By construction we have

$$
\square_{U} \forall \phi \in I\left(\left(\operatorname{Con}^{J}(V) \wedge \square_{V}^{J} \phi\right) \rightarrow \phi \in \mathcal{M}_{V}^{I}\right) .
$$


By the outside big, inside small principle and the formalized deduction theorem we now conclude that

$$
\forall \phi\left(\square_{V} \varphi \rightarrow \square_{U \cup\left\{\operatorname{Con}^{J}(V)\right\}} \varphi^{j}\right)
$$

which, by (10) is nothing but $\left(U \cup\left\{\operatorname{Con}^{J}(V)\right\} \triangleright V\right)$. We refer to [15] where one can see that the necessary induction for this argument is available in $S_{2}^{1}$ and that moving from $\phi \in \mathcal{M}_{V}^{I}$ to $\phi^{j}$ by applying the commutation clauses can be done in p-time.

Using these lemmas we can infer in various occasions the existence of small witnesses to provability. Given two sentences $\alpha$ and $\beta$, it is common practice to denote $(T \cup\{\alpha\}) \triangleright(T \cup\{\beta\})$ by $\alpha \triangleright_{T} \beta$. Moreover, it is common practice to omit theory subscripts in both the interpretation predicate $\triangleright_{T}$ and in the provability predicate $\square_{T}$ and we will do so too. As such, both the arithmetical predicate and modal operator are denoted by the same symbol but the context will always clearly indicate which reading to employ.

Lemma 2.6. For any theory $T$ we have $T \vdash \neg(\alpha \triangleright \neg \gamma) \rightarrow \forall$ Cut $K \diamond\left(\alpha \wedge \square^{K} \gamma\right)$.

Proof. Reason in arbitrary $T$ by contraposition and apply the Henkin construction on a cut.

As a corollary to this lemma, we see that

$$
(A \triangleright B) \rightarrow(\neg(A \triangleright \neg C) \triangleright(B \wedge \square C)) \in \mathbf{I L}(\mathrm{T})
$$

for any $T$ extending $S_{2}^{1}$. It is an open problem to classify the modal principles that hold in any theory extending $S_{2}^{1}$. This paper raises the previously known lower bound.

We formulate some other direct corollaries of the outside-big inside-small principle in the following useful lemma.

Lemma 2.7. Let $T$ be any theory containing $\mathrm{S}_{2}^{1}$. We have that

1. $T \vdash \forall \gamma \in \exists \Sigma_{1}^{b} \forall^{\text {Cut }} J(\exists x \gamma \rightarrow \square \exists x \in J \gamma)$;

2. $T \vdash \sigma \rightarrow \forall{ }^{\text {Cut }} K \square \sigma^{K}$ for any formula $\sigma$ in $\exists \Sigma_{1}^{b}$;

3. $T \vdash \forall \alpha\left(\square \alpha \rightarrow \forall^{\text {Cut }} K \square \square^{K} \alpha\right)$.

One ingredient in proving interpretability principles arithmetically sound, is to find small witnesses. Another ingredient tells us how we can keep these witnesses small. A simple generalization of Pudlák's lemma which was first proved in [6] and tells us how to do so.

Lemma 2.8. Let $T$ be a theory extending $\mathrm{S}_{2}^{1}$.

$$
T \vdash \forall \alpha, \beta \forall^{\text {int }} j\left(j: \alpha \triangleright \beta \rightarrow \forall^{\text {Cut }} I \exists^{\text {Cut }} J j:\left(\alpha \wedge \square^{J} \gamma\right) \triangleright\left(\beta \wedge \square^{I} \gamma\right)\right) .
$$




\subsection{Modal interpretability logics}

When working in interpretability logic, we shall adopt a reading convention that will allow us to omit many brackets. Thus, we say that the strongest binding 'connectives' are $\neg, \square$ and $\diamond$ which all bind equally strong. Next come $\wedge$ and $\vee$, followed by $\triangleright$ and the weakest connective is $\rightarrow$. Thus, for example, $A \triangleright B \rightarrow A \wedge \square C \triangleright B \wedge \square C$ will be short for $(A \triangleright B) \rightarrow((A \wedge \square C) \triangleright(B \wedge \square C))$.

If we do not disambiguate a formula of nested conditionals $(\rightarrow$ or $\triangleright)$, then this should be read as a conjunction. For example, $A \triangleright B \triangleright C$ should be read as $(A \triangleright B) \wedge(B \triangleright C)$ and likewise for implications.

We first define the core logic IL which shall be present in any other interpretability logic. As before, we work in a propositional signature where apart from the classical connectives we have a unary modal operator $\square$ and a binary modal operator $\triangleright$.

Definition 2.9 (IL). The logic IL contains apart from all propositional logical tautologies, all instantiations of the following axiom schemes.

$$
\begin{aligned}
& \mathrm{L} 1 \square(A \rightarrow B) \rightarrow(\square A \rightarrow \square B) \\
& \text { L2 } \square A \rightarrow \square \square A \\
& \text { L3 } \square(\square A \rightarrow A) \rightarrow \square A \\
& \text { J1 } \square(A \rightarrow B) \rightarrow A \triangleright B \\
& \text { J2 }(A \triangleright B) \wedge(B \triangleright C) \rightarrow A \triangleright C \\
& \text { J3 }(A \triangleright C) \wedge(B \triangleright C) \rightarrow A \vee B \triangleright C \\
& \text { J4 } A \triangleright B \rightarrow(\diamond A \rightarrow \diamond B) \\
& \text { J5 } \diamond A \triangleright A
\end{aligned}
$$

The rules of the logic are Modus Ponens (from $A \rightarrow B$ and $A$, conclude $B$ ) and Necessitation (from $A$ conclude $\square A$ ).

It is not hard to see that $\mathbf{I L} \subseteq \mathbf{I L}(\mathrm{All})$. By ILM we denote the logic that arises by adding Montagna's axiom scheme

$$
\mathrm{M}: \quad A \triangleright B \rightarrow A \wedge \square C \triangleright B \wedge \square C
$$

to IL. It follows from our earlier observations that ILM $\subseteq$ IL(T) and the other inclusion can be proven too. A theory $T$ is called $\Sigma_{1}^{0}$-sound if it proves no false $\Sigma_{1}^{0}$-sentences.

Theorem 2.10 (Berarducci [1, Shavrukov [1]). If $T$ is $\Sigma_{1}^{0}$-sound and proves full induction, then $\mathbf{I L}(\mathrm{T})=\mathbf{I L M}$. 
The logic ILP arises by adding the axiom scheme

$$
\mathrm{P}: \quad A \triangleright B \rightarrow \square(A \triangleright B)
$$

to the basic logic IL. If $T$ is finitely axiomatizable it is easy to see that (1) is provably equivalent to a $\Sigma_{1}$ formula so that by provable $\Sigma_{1}$ completeness we see that ILP $\subseteq \mathbf{I L}(\mathrm{T})$ for any finitely axiomatized theory $T$ that proves that exponentiation is a total function. If $T$ can moreover prove the totality of superexponentiation supexp then the inclusion can be reversed too. Here, $\operatorname{supexp}(x)$ is defined as $x \mapsto 2_{x}^{x}$ with $2_{0}^{n}:=n$ and $2_{m+1}^{n}:=2^{\left(2_{m}^{n}\right)}$.

Theorem 2.11 (Visser [14). If $T$ is finitely axiomatizable, proves the totality of supexp, and is $\Sigma_{1}^{0}$-sound, then $\mathbf{I L}(\mathrm{T})=\mathbf{I L P}$.

It follows that $\mathbf{I L} \subseteq \mathbf{I L}(\mathrm{All}) \subseteq(\mathbf{I L P} \cap \mathbf{I L M})$. In this paper we shall focus on these bounds.

\subsection{Relational semantics}

We can equip interpretability logics with a natural relational semantics often referred to as Veltman semantics.

Definition 2.12. A Veltman frame is a triple $\left\langle W, R,\left\{S_{x}\right\}_{x \in W}\right\rangle$ where $W$ is a non-empty set of possible worlds, $R$ a binary relation on $W$ so that $R^{-1}$ is transitive and well-founded. Here, each $S_{x}$ is a binary relations on $x \uparrow$ (where $x \uparrow:=\{y \mid x R y\}$ ). The requirements are that the $S_{x}$ are reflexive and transitive and the restriction of $R$ to $x \uparrow$ is contained in $S_{x}$, that is $R \cap(x \uparrow) \subseteq S_{x}$.

$A$ Veltman model consists of a Veltman frame together with a valuation $V: \operatorname{Prop} \rightarrow \mathcal{P}(W)$ that assigns to each propositional variable $p \in \operatorname{Prop}$ a set of worlds $V(p)$ in $W$ where $p$ is stipulated to be true. This valuation defines a forcing relation $\Vdash \subseteq W \times$ Form telling us which formulas are true at which particular world:

$$
\begin{array}{lll}
x \Vdash \perp & & \text { for no } x \in W ; \\
x \Vdash A \rightarrow B & : \Leftrightarrow \quad x \nVdash A \text { or } x \Vdash B ; \\
x \Vdash \square A & : \Leftrightarrow \quad \forall y(x R y \rightarrow y \Vdash A) ; \\
x \Vdash A \triangleright B & : \Leftrightarrow \quad \forall y\left(x R y \wedge y \Vdash A \rightarrow \exists z\left(y S_{x} z \wedge z \Vdash B\right) .\right.
\end{array}
$$

For a Veltman model $\mathcal{M}=\left\langle W, R,\left\{S_{x}\right\}_{x \in W}, V\right\rangle$, we shall write $\mathcal{M} \models A$ as short for $\forall x \in W \mathcal{M}, x \Vdash A$.

The logic IL is sound and complete with respect to all Veltman models ([3]). Often one is interested in considering all models that can be defined over a frame. Thus, given a frame $\mathcal{F}$ and a valuation $V$ on $\mathcal{F}$ we shall denote the corresponding model by $\langle\mathcal{F}, V\rangle$. A frame condition for a modal formula schema A is a formula $F$ (first or higher-order) in the language $\left\{R,\left\{S_{x}\right\}_{x \in W}\right\}$ so that $\mathcal{F} \models F$ (as a relational structure) if and only if $\forall^{\text {valuation }} V\langle\mathcal{F}, V\rangle \models \mathrm{A}$. 
It is easy to establish that the frame condition for $\mathrm{P}$ is $x R y R z S_{x} u \rightarrow z S_{y} u$ where $x R y R z S_{x} u$ is short for $x R y \wedge y R z \wedge z S_{x} u$. Likewise, it is elementary to see that the frame condition for $\mathrm{M}$ is given by $y S_{x} z R u \rightarrow y R u$. In this paper we shall compute the frame conditions for two new series of principles in IL(All).

Often we shall denote a valuation $V$ directly by the induced forcing relation $\Vdash$. Given a Veltman model $\langle\mathcal{F}, \Vdash\rangle$ we define a $C$-assuring successor — denoted by $R_{\Vdash}^{C}$ - as follows

$$
x R_{\Vdash}^{C} y:=\left(x R y \wedge y \Vdash C \wedge \forall z\left(y S_{x} z \rightarrow z \Vdash C\right)\right) .
$$

\section{A slim hierarchy of principles}

In this section we present a hierarchy of interpretability principles in $\mathbf{I L}(\mathrm{All})$ of growing strength. For a well-behaved sub-hierarchy we shall compute the frame conditions and prove arithmetical soundness. There is no particular 'slimness' inherent to the hierarchy presented here. The main reason for our name is that we tend to depict the frame conditions (see Figure 11) in a slim way as opposed to the depicted frame conditions for the series of principles that we refer to as a broad series of principles (see Figure 2).

Here and in the next section we shall refrain from denoting arithmetical sentences by greek lower-case letters and modal formulas by latin upper-case letters. We will use the latter for both and the context will clearly tell which reading to use.

\subsection{A slim hierarchy}

Let $a_{i}, b_{i}, c_{i}$ and $e_{i}$ denote different propositional variables for $i \in \omega$. Inductively, we define a series of principles as follows.

$$
\begin{aligned}
\mathrm{R}_{0}:= & a_{0} \triangleright b_{0} \rightarrow \neg\left(a_{0} \triangleright \neg c_{0}\right) \triangleright b_{0} \wedge \square c_{0} \\
\mathrm{R}_{2 \mathrm{n}+1}:= & R_{2 n}\left[\neg\left(a_{n} \triangleright \neg c_{n}\right) / \neg\left(a_{n} \triangleright \neg c_{n}\right) \wedge\left(e_{n+1} \triangleright \triangleright a_{n+1}\right) ;\right. \\
& \left.b_{n} \wedge \square c_{n} / b_{n} \wedge \square c_{n} \wedge\left(e_{n+1} \triangleright a_{n+1}\right)\right] \\
\mathrm{R}_{2 \mathrm{n}+2}:= & R_{2 n+1}\left[b_{n} / b_{n} \wedge\left(a_{n+1} \triangleright b_{n+1}\right) ;\right. \\
& \diamond a_{n+1} / \neg\left(a_{n+1} \triangleright \neg c_{n+1}\right) ; \\
& \left.\left(e_{n+1} \triangleright a_{n+1}\right) /\left(e_{n+1} \triangleright a_{n+1}\right) \wedge\left(e_{n+1} \triangleright b_{n+1} \wedge \square c_{n+1}\right)\right]
\end{aligned}
$$


To illustrate how these substitutions work we shall calculate the first five principle schemes.

$$
\begin{aligned}
\mathrm{R}_{0}:= & A_{0} \triangleright B_{0} \rightarrow \neg\left(A_{0} \triangleright \neg C_{0}\right) \triangleright B_{0} \wedge \square C_{0} \\
\mathrm{R}_{1}:= & A_{0} \triangleright B_{0} \rightarrow \neg\left(A_{0} \triangleright \neg C_{0}\right) \wedge\left(E_{1} \triangleright \vee A_{1}\right) \triangleright B_{0} \wedge \square C_{0} \wedge\left(E_{1} \triangleright A_{1}\right) \\
\mathrm{R}_{2}:=\quad & A_{0} \triangleright B_{0} \wedge\left(A_{1} \triangleright B_{1}\right) \rightarrow \neg\left(A_{0} \triangleright \neg C_{0}\right) \wedge\left(E_{1} \triangleright \neg\left(A_{1} \triangleright \neg C_{1}\right)\right) \triangleright \\
& B_{0} \wedge\left(A_{1} \triangleright B_{1}\right) \wedge \square C_{0} \wedge\left(E_{1} \triangleright A_{1}\right) \wedge\left(E_{1} \triangleright B_{1} \wedge \square C_{1}\right) \\
\mathrm{R}_{3}:=\quad & A_{0} \triangleright B_{0} \wedge\left(A_{1} \triangleright B_{1}\right) \rightarrow \\
& \neg\left(A_{0} \triangleright \neg C_{0}\right) \wedge\left(E_{1} \triangleright \neg\left(A_{1} \triangleright \neg C_{1}\right) \wedge\left(E_{2} \triangleright \triangleright A_{2}\right)\right) \triangleright \\
& B_{0} \wedge\left(A_{1} \triangleright B_{1}\right) \wedge \square C_{0} \wedge\left(E_{1} \triangleright A_{1}\right) \wedge\left(E_{1} \triangleright B_{1} \wedge \square C_{1} \wedge\left(E_{2} \triangleright A_{2}\right)\right) \\
\mathrm{R}_{4}:=\quad & A_{0} \triangleright B_{0} \wedge\left(A_{1} \triangleright B_{1} \wedge\left(A_{2} \triangleright B_{2}\right)\right) \rightarrow \\
& \neg\left(A_{0} \triangleright \neg C_{0}\right) \wedge\left(E_{1} \triangleright \neg\left(A_{1} \triangleright \neg C_{1}\right) \wedge\left(E_{2} \triangleright \neg\left(A_{2} \triangleright \neg C_{2}\right)\right)\right) \triangleright \\
& B_{0} \wedge\left(A_{1} \triangleright B_{1} \wedge\left(A_{2} \triangleright B_{2}\right)\right) \wedge \square C_{0} \wedge\left(E_{1} \triangleright A_{1}\right) \wedge \\
& \left(E_{1} \triangleright B_{1} \wedge\left(A_{2} \triangleright B_{2}\right) \wedge \square C_{1} \wedge\left(E_{2} \triangleright A_{2}\right) \wedge\left(E_{2} \triangleright B_{2} \wedge \square C_{2}\right)\right)
\end{aligned}
$$

It is easy to see that the hierarchy defines a series of principles of increasing strength as expressed by the following lemma.

Lemma 3.1. For each natural number $n$ we have that $\mathrm{ILR}_{\mathrm{n}+1} \vdash \mathrm{R}_{\mathrm{n}}$.

Proof. By an easy case distinction. We see that $\vdash_{\mathrm{IL}} \mathrm{R}_{2 n+1} \rightarrow \mathrm{R}_{2 n}$ by choosing $E_{n+1}:=\diamond \top$ and $A_{n+1}:=\top$. To see that $\vdash_{\mathrm{IL}} \mathrm{R}_{2 n+2} \rightarrow \mathrm{R}_{2 n+1}$ we choose $C_{n+1}:=\top$ and $B_{n+1}:=A_{n+1}$.

Thus, to understand the hierarchy well, it suffices to study a well-behaved co-final subsequence of it. To this end we define the following hierarchy.

For any $n \geq 0$ we define schemata $\mathrm{X}_{n}, \mathrm{Y}_{n}$ and $\mathrm{Z}_{n}$ as follows.

$$
\begin{aligned}
\mathrm{X}_{0} & =a_{0} \triangleright b_{0} ; \\
\mathrm{Y}_{0} & =\neg\left(a_{0} \triangleright \neg c_{0}\right) ; \\
\mathrm{Z}_{0} & =b_{0} \wedge \square c_{0} ; \\
\mathrm{X}_{n+1} & =a_{n+1} \triangleright b_{n+1} \wedge\left(\mathrm{X}_{n}\right) ; \\
\mathrm{Y}_{n+1} & =\neg\left(a_{n+1} \triangleright \neg c_{n+1}\right) \wedge\left(e_{n+1} \triangleright \mathrm{Y}_{n}\right) ; \\
\mathrm{Z}_{n+1} & =b_{n+1} \wedge\left(\mathrm{X}_{n}\right) \wedge \square c_{n+1} \wedge\left(e_{n+1} \triangleright a_{n}\right) \wedge\left(e_{n+1} \triangleright \mathrm{Z}_{n}\right) .
\end{aligned}
$$

For any $n \geq 0$ define

$$
\widetilde{\mathrm{R}}_{n}=\mathrm{X}_{n} \rightarrow \mathrm{Y}_{n} \triangleright \mathrm{Z}_{n} .
$$

To see how this proceeds, let us evaluate the first couple of schematic instances:

$$
\begin{aligned}
& \widetilde{\mathrm{R}}_{0}:=A_{0} \triangleright B_{0} \rightarrow \neg\left(A_{0} \triangleright \neg C_{0}\right) \triangleright B_{0} \wedge \square C_{0} ; \\
& \widetilde{\mathrm{R}}_{1}:=A_{1} \triangleright B_{1} \wedge\left(A_{0} \triangleright B_{0}\right) \rightarrow \\
& \neg\left(A_{1} \triangleright \neg C_{1}\right) \wedge\left(E_{1} \triangleright \neg\left(A_{0} \triangleright \neg C_{0}\right)\right) \triangleright \\
& B_{1} \wedge\left(A_{0} \triangleright B_{0}\right) \wedge \square C_{1} \wedge\left(E_{1} \triangleright A_{0}\right) \wedge\left(E_{1} \triangleright B_{0} \wedge \square C_{0}\right) ; \\
& \widetilde{\mathrm{R}}_{2}:=A_{2} \triangleright B_{2} \wedge\left(A_{1} \triangleright B_{1} \wedge\left(A_{0} \triangleright B_{0}\right)\right) \rightarrow \\
& \neg\left(A_{2} \triangleright \neg C_{2}\right) \wedge\left(E_{2} \triangleright \neg\left(A_{1} \triangleright \neg C_{1}\right) \wedge\left(E_{1} \triangleright \neg\left(A_{0} \triangleright \neg C_{0}\right)\right)\right) \triangleright \\
& B_{2} \wedge\left(A_{1} \triangleright B_{1} \wedge\left(A_{0} \triangleright B_{0}\right)\right) \wedge \square C_{2} \wedge\left(E_{2} \triangleright A_{1}\right) \wedge \\
& \left(E_{2} \triangleright B_{1} \wedge\left(A_{0} \triangleright B_{0}\right) \wedge \square C_{1} \wedge\left(E_{1} \triangleright A_{0}\right) \wedge\left(E_{1} \triangleright B_{0} \wedge \square C_{0}\right)\right) \text {; }
\end{aligned}
$$


It is clear that the $\widetilde{\mathrm{R}}_{k}$ hierarchy is directly related to the $\mathrm{R}_{k}$ hierarchy:

Lemma 3.2. For each natural number $k$ we have $\mathrm{R}_{2 k}:=\widetilde{\mathrm{R}}_{k}\left[\mathbb{X}_{i} / \mathbb{X}_{k-i} ; E_{i} / E_{k+1-i}\right]$, where $\mathbb{X} \in\{A, B, C\}$.

Proof. By visual inspection we see that it holds for $k=0,1$. It is proven in full generality by an easy induction. To prove the lemma, it is best to consider the place-holders like $A_{i}$ etc. as propositional variables since otherwise in principle, for example, $A_{i}$ could contain $E_{i}$ as a subformula.

For the remainder of this section, we shall focus on the $\widetilde{R}_{k}$ hierarchy and begin by computing a collection of frame conditions.

\subsection{Frame conditions}

For any $n \geq 0$ we define a ternary relation $\mathcal{G}_{n}(x, y, z)$ on Veltman-frames as follows.

$$
\begin{aligned}
\mathcal{G}_{0}(x, y, z) & =\forall u\left(z R u \Rightarrow y S_{x} u\right) \\
\mathcal{G}_{n+1}(x, y, z) & =\forall u\left(z R u \Rightarrow y S_{x} u \wedge \forall v\left(u S_{x} v \Rightarrow \mathcal{G}_{n}(z, u, v)\right) .\right.
\end{aligned}
$$

For every $n \geq 0$ we define the first-order frame condition $\mathcal{F}_{n}$ as follows.

$$
\mathcal{F}_{n}=\forall w, x, y, z\left(w R x R y S_{w} z \Rightarrow \mathcal{G}_{n}(x, y, z)\right) .
$$

The main result of this subsection is that $\mathcal{F}_{2 n}$ is the frame correspondence of $\widetilde{\mathrm{R}}_{n}$. For $n=0$ this has been established in [4]. It is easy to see that $\mathcal{G}_{n+1}(x, y, z)$ implies $\mathcal{G}_{n}(x, y, z)$ so that $\mathcal{F}_{n+1}$ also implies $\mathcal{F}_{n}$. The frame conditions $\mathcal{F}_{k}$ are depicted in Figure 1 for the first three values of $k$.

In what follows we let $F=\langle W, R, S\rangle$ be an arbitrary Veltman-frame. With a forcing relation $\Vdash$ we will always mean a forcing relation on $F$. For our convenience we define

$$
A_{-1} \equiv \mathrm{X}_{-1} \equiv \mathrm{Z}_{-1} \equiv \mathrm{\top} .
$$

Before we can prove a frame correspondence we first need a technical lemma.

Lemma 3.3. For all $k \geq 0$ and all $x, y, z \in W$. If $\mathcal{G}_{2 k}(x, y, z)$ then for any forcing relation $\Vdash$ for which

$$
x \Vdash \mathrm{Y}_{k} \quad \text { and } \quad x R_{\Vdash}^{C_{k}} y \quad \text { and } \quad z \Vdash \mathrm{X}_{k-1},
$$

we also have

$$
z \Vdash \square C_{k} \wedge\left(E_{k} \triangleright A_{k-1}\right) \wedge\left(E_{k} \triangleright \mathrm{Z}_{k-1}\right) .
$$

Proof. We shall write $x R^{C_{k}} y$ as short for $x R_{\Vdash}^{C_{k}} y$ and prove the claim by induction on $k$. With the convention that $A_{-1} \equiv \mathrm{Z}_{-1} \equiv \top$ the lemma is trivial for 

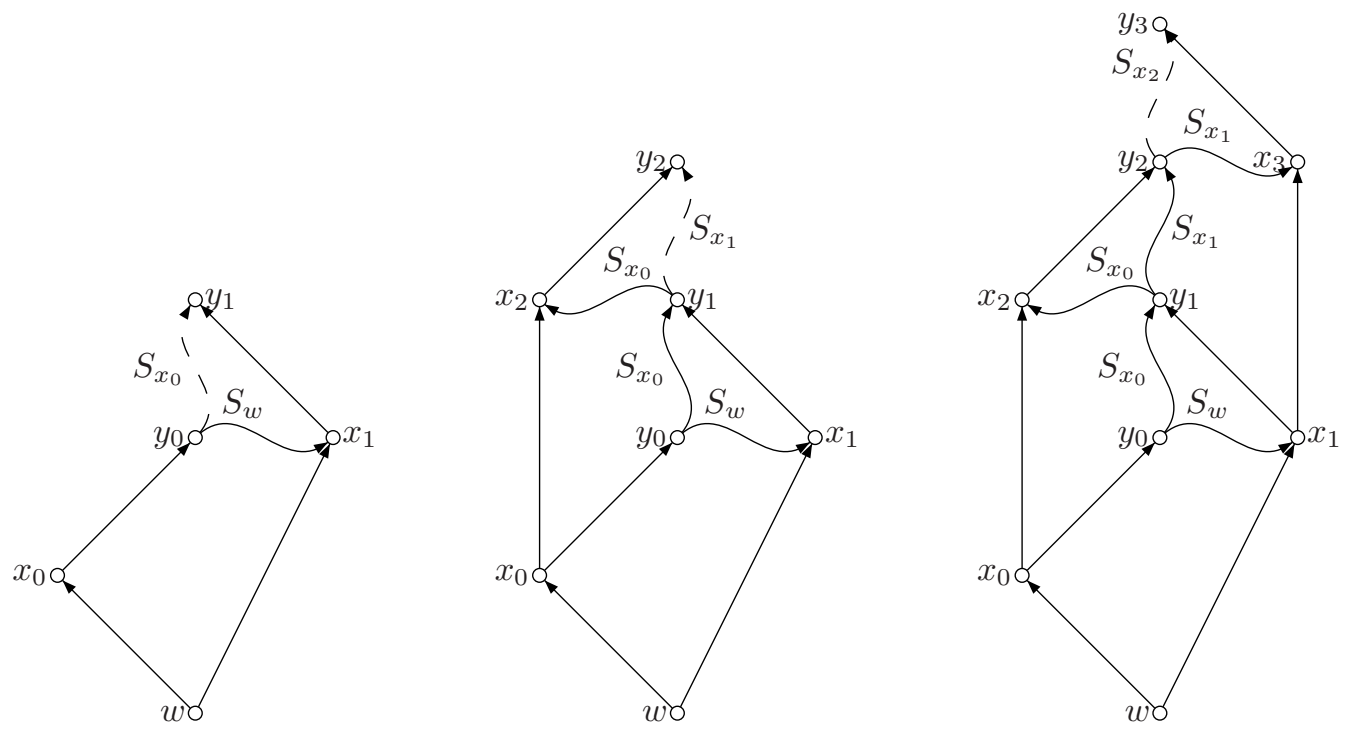

Figure 1: From left to right we have depicted $\mathcal{F}_{0}$ to $\mathcal{F}_{2}$. Since $\mathcal{F}_{k+1}$ implies $\mathcal{F}_{k}$ we have only depicted the content of $\mathcal{F}_{k+1}$ which is new w.r.t. $\mathcal{F}_{k}$. As such we should read the pictures as: "if all un-dashed relations are as in the picture, then also the dashed relation should be present".

$k=0$. So assume $k>0$. Let $\Vdash$ be a forcing relation and take $x, y$ and $z$ such that

$$
\begin{aligned}
& x \Vdash \neg\left(A_{k} \triangleright \neg C_{k}\right) \wedge\left(E_{k} \triangleright \mathrm{Y}_{k-1}\right), \\
& x R^{C_{k}} y, \\
& z \Vdash \mathrm{X}_{k-1}, \\
& \mathcal{G}_{2 k}(x, y, z) .
\end{aligned}
$$

Take an arbitrary $u \in W$ with $z R u$. By (7) we have $y S_{x} u$ and thus by (5D) we have $u \Vdash C_{k}$. This shows $z \Vdash \square C_{k}$.

To show that also the other two conjuncts hold at $z$ assume that $u \Vdash E_{k}$. By (4) we find some $v$ with $u S_{x} v$ and

$$
v \Vdash \mathrm{Y}_{k-1} .
$$

In order to show $z \Vdash E_{k} \triangleright A_{k-1}$ we have to find some $a$ with $u S_{z} a \Vdash A_{k-1}$. We note that $\mathrm{Y}_{k-1}$ implies $\diamond A_{k-1}$ thus there exists some $a$ with $v R a \Vdash A_{k-1}$. By (77) we have $\mathcal{G}_{2 k-1}(z, u, v)$ and thus $u S_{z} a$. 
In order to show that also $z \Vdash E_{k} \triangleright Z_{k-1}$ we have to find some $b$ with $u S_{z} b \Vdash \mathrm{Z}_{k-1}$. We just used that $\mathrm{Y}_{k-1}$ implies $\diamond A_{k-1}$, but we note that $\mathrm{Y}_{k-1}$ implies the stronger statement that $\neg\left(A_{k-1} \triangleright \neg C_{k-1}\right)$. Thus there exists some $a$ with $a \Vdash A_{k-1}$ and

$$
v R^{C_{k-1}} a .
$$

As above, by (77) we have $\mathcal{G}_{2 k-1}(z, u, v)$ and thus $u S_{z} a$ and $z R a$. By (6) there exists a $b$ with $a S_{z} b$ and

$$
b \Vdash B_{k-1} \wedge\left(\mathrm{X}_{k-2}\right) .
$$

Since $u S_{z} a$ whence also $u S_{z} b$ holds, we will be done if we show that $b \Vdash Z_{k-1}$. To show that the remaining conjuncts of $Z_{k-1}$ hold at $b$ (that is $b \Vdash \square C_{k-1} \wedge$ $\left.\left(E_{k-1} \triangleright A_{k-2}\right) \wedge\left(E_{k-1} \triangleright \mathbf{Z}_{k-2}\right)\right)$ simply observe that $\mathcal{G}_{2 k-2}(v, a, b)$ and use (8), (9) and (10) to invoke the (IH) on $v, a$ and $b$.

Corollary 3.4. If $F \models \mathcal{F}_{2 k}$, then $F \models \widetilde{\mathrm{R}}_{k}$.

Proof. Fix a forcing relation $\Vdash$ and let $w, x \in W$ such that $w \Vdash \mathbf{X}_{k}$ and $w R x \Vdash$ $\mathrm{Y}_{k}$. Then for some $y$ we have $x R^{C_{k}} y \Vdash A_{k}$. Thus there exists $z$ with $y S_{w} z$ and

$$
z \Vdash B_{k} \wedge\left(\mathrm{X}_{k-1}\right)
$$

(recall $\mathrm{X}_{-1} \equiv \top$ ). Since $F \models \mathcal{F}_{2 k}$ we have $\mathcal{G}_{2 k}(x, y, z)$. Thus by Lemma 3.3 we get

$$
z \Vdash \square C_{k} \wedge\left(E_{k} \triangleright A_{k-1}\right) \wedge\left(E_{k} \triangleright \mathrm{Z}_{k-1}\right) .
$$

Combining (11) and (12) gives $z \Vdash \mathrm{Z}_{k}$.

The reversal of this corollary is again preceded by a technical lemma. We shall denote by $\boldsymbol{a}_{k}, \boldsymbol{b}_{k}, \boldsymbol{c}_{k}$, and $\boldsymbol{e}_{k}$, propositional variables that shall play the role of the $A_{k}, B_{k}, C_{k}$ and $E_{k}$ respectively in the principles $\widetilde{\mathrm{R}}_{n}$. Likewise, by $\overline{\mathrm{X}}_{k}$ we shall denote the formula that arises by substituting $\boldsymbol{a}_{j}$ for $A_{j}$ in $\mathrm{X}_{k}$ and $\boldsymbol{b}_{j}$ for $B_{j}$. The formulas $\bar{Y}_{k}$ and $\bar{Z}_{k}$ are defined similarly.

Lemma 3.5. For any $k \geq 0$ and all $x, y, z \in W$. If for all forcing relations $\Vdash$ for which

$$
x \Vdash \overline{\mathrm{Y}}_{k} \text { and } x R_{\Vdash}^{c_{k}} y \text { and } z \Vdash \overline{\mathrm{X}}_{k-1}
$$

we also have

$$
z \Vdash \square c_{k} \wedge\left(\boldsymbol{e}_{k} \triangleright \boldsymbol{a}_{k-1}\right) \wedge\left(\boldsymbol{e}_{k} \triangleright \overline{\mathrm{Z}}_{k-1}\right),
$$

then $\mathcal{G}_{2 k}(x, y, z)$.

Proof. Induction on $k$. Let $x, y, z \in W$ and assume the conditions of the lemma. Unfolding the definition of $\mathcal{G}_{2 k}(x, y, z)$ shows us that we have to show that

1. for all $u$ with $z R u$ we have $y S_{x} u$ $(k \geq 0) ;$

2. and for all $v$ and $a$ with $u S_{x} v$ and $v R a$ we have $u S_{z} a$

$(k>0)$;

3. and for all $b$ with $a S_{z} b$ we have $\mathcal{G}_{2(k-1)}(v, a, b)$ $(k>0)$. 
We will show 1 and 2 'by hand' and invoke the $(\mathrm{IH})$ for 3 In each of the three cases we will choose similar but different forcing relations $\Vdash$.

We first show 1 So let $z R u$. Define

$$
w \Vdash \boldsymbol{c}_{k} \Leftrightarrow y S_{x} w \quad \text { and } \quad w \Vdash \boldsymbol{a}_{k} \Leftrightarrow w=y .
$$

And let all the other variables be false everywhere. Then $x R_{\Perp}^{c_{k}} y$ and $x \Vdash$ $\neg\left(\boldsymbol{a}_{k} \triangleright \neg \boldsymbol{c}_{k}\right)$. Since none of the $\boldsymbol{e}_{i}$ nor $\boldsymbol{a}_{j}$ with $j \neq k$ holds anywhere in the model, we trivially have $x \Vdash \bar{Y}_{k}$ and $z \Vdash \bar{X}_{k-1}$ and thus according to the conditions of the lemma in particular $z \Vdash \square c_{k}$. By definition of $\Vdash$ we thus have $y S_{x} u$ which proves 1 . Note that for $k=0$ we only have to look after 1 hence we have now dealt with the base case of our induction.

Now we continue to show 2 assuming $k>0$. Choose any $v$ and $a$ with $u S_{x} v$ and $v R a$. As above define

$$
w \Vdash \boldsymbol{c}_{k} \Leftrightarrow y S_{x} w \quad \text { and } \quad w \Vdash \boldsymbol{a}_{k} \Leftrightarrow w=y .
$$

We now also define

$$
\begin{array}{ll}
w \Vdash \boldsymbol{e}_{k} \Leftrightarrow w=u & \text { and, } \\
w \Vdash \boldsymbol{a}_{k-1} \Leftrightarrow w=a \Leftrightarrow w \Vdash \boldsymbol{b}_{k-1} & \text { and, } \\
w \Vdash \boldsymbol{c}_{k-1} \Leftrightarrow a S_{z} w . &
\end{array}
$$

Let all the other propositional variables be false everywhere. Now $v \Vdash \bar{Y}_{k-1}$ and thus $x \Vdash \bar{Y}_{k}$. It is not hard to see that we also have $z \Vdash \bar{X}_{k-1}$ and thus according to the condition of the lemma we have in particular $z \Vdash \boldsymbol{e}_{k} \triangleright \boldsymbol{a}_{k-1}$. Since $z R u \Vdash \boldsymbol{e}_{k}$ there must be an $a^{\prime}$ with $u S_{z} a^{\prime} \Vdash \boldsymbol{a}_{k-1}$. Since $a$ is the only world that forces $\boldsymbol{a}_{k-1}$ we must have $u S_{z} a$.

To finish and show 3 choose $b$ such that $a S_{z} b$. We want to show that $\mathcal{G}_{2(k-1)}(v, a, b)$. Invoking the $(\mathrm{IH})$ it is enough to show that for any forcing relation $\Vdash$ for which

$$
v \Vdash \overline{\mathrm{Y}}_{k-1}, \quad \text { and } \quad v R_{\Vdash}^{c_{k-1}} a \text { and } \quad b \Vdash \overline{\mathrm{X}}_{k-2},
$$

we also have

$$
b \Vdash\left(\boldsymbol{e}_{k-1} \triangleright \boldsymbol{a}_{k-2}\right) \wedge\left(\boldsymbol{e}_{k-1} \triangleright \overline{\mathrm{Z}}_{k-2}\right) \wedge \square \boldsymbol{c}_{k-1} .
$$

Our strategy in proving this is as follows. We slightly tweak $\Vdash$ to obtain $\Vdash^{\prime}$. This $\Vdash^{\prime}$ is similar to $\Vdash$ in that (13) still holds and moreover

$b \Vdash A \Leftrightarrow b \Vdash^{\prime} A$ for subformulas $A$ of $\left(\boldsymbol{e}_{k-1} \triangleright \boldsymbol{a}_{k-2}\right) \wedge\left(\boldsymbol{e}_{k-1} \triangleright \bar{Z}_{k-2}\right) \wedge \square \boldsymbol{c}_{k-1}$.

However, it is (possibly) different in that we now know that $x \Vdash^{\prime} \bar{Y}_{k}$, and $x R_{R^{\prime}}^{c_{k}} y$ and, $z \Vdash^{\prime} \bar{X}_{k-1}$ so that we may apply the main assumption of the lemma to $\Vdash^{\prime}$ concluding $z \Vdash^{\prime} \square \boldsymbol{c}_{k} \wedge\left(\boldsymbol{e}_{k} \triangleright \boldsymbol{a}_{k-1}\right) \wedge\left(\boldsymbol{e}_{k} \triangleright \overline{\mathbf{Z}}_{k-1}\right)$. The latter will help us conclude (14).

Thus we consider an arbitrary forcing relation $\Vdash$ that satisfies (13). We modify $\Vdash$ to obtain $\Vdash^{\prime}$ such that it satisfies 


$$
\begin{array}{lll}
w \Vdash^{\prime} \boldsymbol{a}_{k} & \Leftrightarrow & w=y ; \\
w \Vdash^{\prime} \boldsymbol{e}_{k} & \Leftrightarrow & =u ; \\
w \Vdash^{\prime} \boldsymbol{c}_{k} & \Leftrightarrow & \Leftrightarrow S_{x} w ; \\
w \Vdash^{\prime} \boldsymbol{a}_{k-1} & \Leftrightarrow & w=a ; \\
w \Vdash^{\prime} \boldsymbol{b}_{k-1} & \Leftrightarrow & w=b .
\end{array}
$$

Apart from these modifications, $\Vdash^{\prime}$ will coincide with $\Vdash$. It is a straightforward check to see that we have (13) for $\Vdash^{\prime}$ and that moreover (15) holds. In addition, by the definition of $\vdash^{\prime}$ we now also have

$$
x \Vdash^{\prime} \overline{\mathrm{Y}}_{k} \text { and } \quad x R_{\Vdash^{\prime}}^{c_{k}} y \text { and } z \Vdash^{\prime} \overline{\mathrm{X}}_{k-1}
$$

Thus, we see that $\Vdash^{\prime}$ satisfies the antecedent of the condition of the lemma. Consequently, we have $z \Vdash^{\prime} \boldsymbol{e}_{k} \triangleright \bar{Z}_{k-1}$. Since $z R u \Vdash^{\prime} \boldsymbol{e}_{k}$, there must exist some $b^{\prime}$ with $u S_{z} b^{\prime} \Vdash^{\prime} \bar{Z}_{k-1}$. But now, since $\boldsymbol{b}_{k-1}$ is a conjunct of $\bar{Z}_{k-1}$ and $b$ is the only world that $\Vdash^{\prime}$-forces $\boldsymbol{b}_{k-1}$, we must have $b \Vdash^{\prime} \bar{Z}_{k-1}$. In particular, we conclude $b \Vdash^{\prime}\left(\boldsymbol{e}_{k-1} \triangleright \boldsymbol{a}_{k-2}\right) \wedge\left(\boldsymbol{e}_{k-1} \triangleright \overline{\mathbf{Z}}_{k-2}\right) \wedge \square \boldsymbol{c}_{k-1}$; by (15) the same holds for $\Vdash$ and we are done.

Putting this all together gives us the frame correspondence for $\widetilde{R}_{k}$.

Theorem 3.6. For any Veltman frame $F$ and any natural number $k \geq 0$ we have

$$
F \models \mathcal{F}_{2 k} \Longleftrightarrow F \models \widetilde{\mathrm{R}}_{k} \Longleftrightarrow F \models \mathrm{R}_{2 k}
$$

Proof. The second equivalence is a direct consequence of Lemma 3.2 so we focus on the first equivalence.

The $\Rightarrow$ direction is just Corollary 3.4. For the other direction, fix some $k$, assume that $F \models \widetilde{\mathrm{R}}_{k}$ and let $w R x R y S_{w} z$. We have to show that $\mathcal{G}_{2 k}(x, y, z)$. Now consider any forcing relation $\Vdash$ that satisfies $x R_{\Vdash}^{c_{k}} y$, and $x \Vdash \bar{Y}_{k}$ and, $z \Vdash \overline{\mathrm{X}}_{k-1}$. By Lemma 3.5 it is enough to show that

$$
z \Vdash \square c_{k} \wedge\left(e_{k} \triangleright \boldsymbol{a}_{k-1}\right) \wedge\left(\boldsymbol{e}_{k} \triangleright \bar{Z}_{k-1}\right) .
$$

Now consider a forcing relation $\Vdash^{\prime}$ where $\Vdash^{\prime}$ is like $\Vdash$ except that

$$
v \Vdash^{\prime} \boldsymbol{a}_{k} \Leftrightarrow v=y \quad \text { and } \quad v \Vdash^{\prime} \boldsymbol{b}_{k} \Leftrightarrow v=z .
$$

Notice that $x R_{\Perp^{\prime}}^{c_{k}} y$ and thus also $x \Vdash^{\prime} \bar{Y}_{k}$. But now we have $w \Vdash^{\prime} \overline{\mathrm{X}}_{k}$ as well and thus $w \Vdash^{\prime} \bar{Y}_{k} \triangleright \bar{Z}_{k}$. Thus there must be some $z^{\prime}$ with $x S_{w} z^{\prime} \Vdash \bar{Z}_{k}$. Since $\boldsymbol{b}_{k}$ is a conjunct of $\bar{Z}_{k}$ and $z$ is the only world where $\boldsymbol{b}_{k}$ is forced we must have $z \Vdash^{\prime} \bar{Z}_{k}$. Since $\square \boldsymbol{c}_{k} \wedge\left(\boldsymbol{e}_{k} \triangleright \boldsymbol{a}_{k-1}\right) \wedge\left(\boldsymbol{e}_{k} \triangleright \bar{Z}_{k-1}\right)$ does not involve $\boldsymbol{a}_{k}$ nor $\boldsymbol{b}_{k}$ we have (17).

\subsection{Arithmetical soundness}

Via a series of lemmata we shall prove Theorem 3.7 to the effect that the hierarchy $\left\{\mathrm{R}_{i}\right\}_{i \in \omega}$ is arithmetically sound in any reasonable arithmetical theory. 
Theorem 3.7. Each of the $\mathrm{R}_{i}$ is arithmetically sound in any theory extending $\mathrm{S}_{2}^{1}$.

It is sufficient to prove that each of the $R_{2 m}$ is arithmetically sound in any reasonable arithmetical theory whence we shall focus on the principles $\widetilde{R}_{i}$. We shall first exhibit a soundness proof of $\widetilde{R}_{1}$ and then indicate how this is generalized to the rest of the hierarchy. Before proving the arithmetical soundness of $\widetilde{R}_{1}$ we first need to prove some auxiliary lemmas.

Lemma 3.8. Let $T$ be any theory extending $\mathrm{S}_{2}^{1}$. We have that for any arithmetical sentences $E_{1}, A_{0}, B_{0}$ and $C_{0}$ that

$$
T \vdash E_{1} \triangleright \neg\left(A_{0} \triangleright \neg C_{0}\right) \rightarrow \exists^{\text {Cut }} J \square\left(E_{1} \rightarrow \forall^{\text {Cut }} K \in J \diamond^{J}\left(A_{0} \wedge \square^{K} C_{0}\right)\right) .
$$

Proof. Reason in $T$ and assume $E_{1} \triangleright \neg\left(A_{0} \triangleright \neg C_{0}\right)$. Note that by Lemma 2.6 we have $E_{1} \triangleright \forall{ }^{\text {Cut }} K \diamond\left(A_{0} \wedge \square^{K} C_{0}\right)$. Consequently, by Pudlák's Lemma, which is our Lemma 2.2. we get $\exists J\left(E_{1} \wedge \exists^{\text {Cut }} K \in J \square^{J} \neg\left(A_{0} \wedge \square^{K} C_{0}\right) \triangleright \perp\right)$. But this is provably the same as $\exists^{\text {Cut }} J \square\left(E_{1} \rightarrow \forall^{\text {Cut }} K \in J \diamond^{J}\left(A_{0} \wedge \square^{K} C_{0}\right)\right)$ as was to be shown.

Lemma 3.9. Let $T$ be any theory extending $\mathrm{S}_{2}^{1}$. We have that for any arithmetical sentences $E_{1}, A_{0}, B_{0}$ and $C_{0}$ that

$$
T \vdash \exists^{\text {Cut }} J \square\left(E_{1} \rightarrow \forall^{\text {Cut }} K \in J \diamond^{J}\left(A_{0} \wedge \square^{K} C_{0}\right)\right) \rightarrow E_{1} \triangleright A_{0} .
$$

Proof. Reason in T. From the assumption we get in particular that

$$
\exists^{\text {Cut }} J \square\left(E_{1} \rightarrow \diamond^{J} A_{0}\right)
$$

so that

$$
\exists^{\text {Cut }} J E_{1} \triangleright \diamond^{J} A_{0} \triangleright A_{0} .
$$

Lemma 3.10. Let $T$ be any theory extending $\mathrm{S}_{2}^{1}$. Then, for any arithmetical sentences $E_{1}, A_{0}, B_{0}$ and $C_{0}$ we have that

$T \vdash\left(A_{0} \triangleright B_{0}\right) \wedge \exists^{\text {Cut }} J \square\left(E_{1} \rightarrow \forall^{\text {Cut }} K \in J \diamond^{J}\left(A_{0} \wedge \square^{K} C_{0}\right)\right) \rightarrow E_{1} \triangleright B_{0} \wedge \square C_{0}$.

Proof. Reasoning in $T$ we get from

$$
\exists^{\text {Cut }} J \square\left(E_{1} \rightarrow \forall^{\text {Cut }} K \in J \diamond^{J}\left(A_{0} \wedge \square^{K} C_{0}\right)\right)
$$

that $\forall^{\text {Cut }} K\left(E_{1} \triangleright A_{0} \wedge \square^{K} C_{0}\right)$. We combine this with

$$
A_{0} \triangleright B_{0} \rightarrow \exists^{\mathrm{Cut}} J\left(A_{0} \wedge \square^{J} C_{0} \triangleright B_{0} \wedge \square C_{0}\right)
$$

to conclude $E_{1} \triangleright B_{0} \wedge \square C_{0}$.

With these technical lemmas we can prove soundness of $\widetilde{R}_{1}$. 
Lemma 3.11. Let $T$ be any theory extending $\mathrm{S}_{2}^{1}$. We have that for any arithmetical sentences $E_{1}, A_{1}, B_{1}, A_{0}, B_{0}$ and $C_{0}$ that

$$
\begin{aligned}
& T \vdash A_{1} \triangleright B_{1} \wedge\left(A_{0} \triangleright B_{0}\right) \rightarrow \\
& \neg\left(A_{1} \triangleright \neg C_{1}\right) \wedge\left(E_{1} \triangleright \neg\left(A_{0} \triangleright \neg C_{0}\right)\right) \triangleright \\
& B_{1} \wedge\left(A_{0} \triangleright B_{0}\right) \wedge \square C_{1} \wedge\left(E_{1} \triangleright A_{0}\right) \wedge\left(E_{1} \triangleright B_{0} \wedge \square C_{0}\right) .
\end{aligned}
$$

Proof. We reason in $T$. To begin, we observe that by Pudlák's Lemma we have

$$
A_{1} \triangleright B_{1} \wedge\left(A_{0} \triangleright B_{0}\right) \rightarrow \exists^{\text {Cut }} K\left(A_{1} \wedge \sigma^{K} \triangleright B_{1} \wedge \sigma \wedge\left(A_{0} \triangleright B_{0}\right)\right)
$$

holds for any $\sigma \in \Sigma_{1}$.

Next, using our new technical lemma and Lemma 2.6 we get

$$
\begin{array}{ll}
\neg\left(A_{1} \triangleright \neg C_{1}\right) \wedge\left(E_{1} \triangleright \neg\left(A_{0} \triangleright \neg C_{0}\right)\right) & \rightarrow \\
\forall^{\text {Cut }} K \diamond\left(A_{1} \wedge \square^{K} C_{1}\right) \wedge \exists \text { Cut } J \square\left(E_{1} \rightarrow \forall^{\text {Cut }} L \in J \diamond^{J}\left(A_{0} \wedge \square^{L} C_{0}\right)\right) & \rightarrow \\
\forall^{\text {Cut }} K \diamond\left(A_{1} \wedge \square^{K} C_{1} \wedge \exists^{\text {Cut }} J \in K \square^{K}\left(E_{1} \rightarrow \forall^{\text {Cut }} L \in J \diamond^{J}\left(A_{0} \wedge \square^{L} C_{0}\right)\right)\right) . &
\end{array}
$$

The last step is due to the principle of outside-big inside-small (Lemma 2.4) and allows us to conclude

$$
\begin{aligned}
\forall^{\text {Cut }} K\left(\neg\left(A_{1} \triangleright \neg C_{1}\right) \wedge\left(E_{1} \triangleright \neg\left(A_{0} \triangleright \neg C_{0}\right)\right) \triangleright\right. \\
\left.A_{1} \wedge \square^{K} C_{1} \wedge \exists^{\text {Cut }} J \in K \square^{K}\left(E_{1} \rightarrow \forall^{\text {Cut }} L \in J \diamond^{J}\left(A_{0} \wedge \square^{L} C_{0}\right)\right)\right) .
\end{aligned}
$$

We can combine this with the particular cut $K$ from (18) to obtain

$$
\begin{aligned}
A_{1} \triangleright B_{1} \wedge\left(A_{0} \triangleright B_{0}\right) \rightarrow \neg\left(A_{1} \triangleright \neg C_{1}\right) \wedge\left(E_{1} \triangleright \neg\left(A_{0} \triangleright \neg C_{0}\right)\right) \triangleright \\
B_{1} \wedge\left(A_{0} \triangleright B_{0}\right) \wedge \square C_{1} \wedge \exists^{\text {Cut }} J \square\left(E_{1} \rightarrow \forall^{\text {cut }} L \in J \diamond^{J}\left(A_{0} \wedge \square^{L} C_{0}\right)\right) .
\end{aligned}
$$

(Note that $\square^{K} C_{1} \wedge \exists^{\text {Cut }} J \in K \square^{K}\left(E_{1} \rightarrow \forall^{\text {Cut }} L \in J \diamond^{J}\left(A_{0} \wedge \square^{L} C_{0}\right)\right)$ is equivalent to an $\exists \Sigma_{1}^{b}$ sentence relativized to $K$.) Our technical lemmas 3.9 and 3.10 tell us that

$$
\begin{array}{r}
\left(A_{0} \triangleright B_{0}\right) \wedge \exists^{\text {Cut }} J \square\left(E_{1} \rightarrow \forall^{\text {Cut }} L \in J \diamond^{J}\left(A_{0} \wedge \square^{L} C_{0}\right)\right) \rightarrow \\
\left(E_{1} \triangleright A_{0}\right) \wedge\left(E_{1} \triangleright B_{0} \wedge \square C_{0}\right)
\end{array}
$$

and we are done.

The soundness proofs for $\widetilde{\mathrm{R}}_{k}$ are essentially not much different. We shall indicate where the soundness proof for $\widetilde{R}_{1}$ needs to be modified and begin with modifications of the technical lemmas.

However, first we must inductively define a series of important formulas. In our definition we work with more variables than actually needed. However, we have chosen to do so since our variables can be interpreted as numbers or as formulas and we wish to avoid expressions like $\forall^{\text {Cut }} J \square \exists J \in J \phi$.

$$
\begin{aligned}
\mathcal{H}_{1} & :=\exists^{\text {Cut }} J_{1} \square\left(E_{1} \rightarrow \forall^{\text {Cut }} K_{1} \in J_{1} \diamond^{J_{1}}\left(A_{0} \wedge \square^{K_{1}} C_{0}\right)\right) ; \\
\mathcal{H}_{k+1} & :=\exists^{\text {Cut }} J_{k+1} \square\left(E_{k+1} \rightarrow \forall^{\text {Cut }} K_{k+1} \in J_{k+1} \diamond^{J_{k+1}}\left(A_{k} \wedge \square^{K_{k+1}} C_{k} \wedge \mathcal{H}_{k}^{K_{k+1}}\right)\right) .
\end{aligned}
$$


It is easy to see that for each $k>0$ the formula $\mathcal{H}_{k}$ is an $\exists \Sigma_{1}^{b}$ formula. The next lemmas show us that $\mathcal{H}_{k+1}$ are $\exists \Sigma_{1}^{b}$ consequences of the $\Sigma_{3}$ statements $E_{k+1} \triangleright Y_{k}$ which contain all the essential information for proving soundness. First we prove a simple modification of Lemma 3.8 .

Lemma 3.12. Let $T$ be any theory extending $\mathrm{S}_{2}^{1}$. For any arithmetical sentences $E_{1}, A_{0}, B_{0}$ and $C_{0}$ and for any $\exists \Sigma_{1}^{b}$ formula $\sigma$ we have

$T \vdash E_{1} \triangleright \neg\left(A_{0} \triangleright \neg C_{0}\right) \wedge \sigma \rightarrow \exists^{\text {Cut }} J \square\left(E_{1} \rightarrow \forall^{\text {Cut }} K \in J \diamond^{J}\left(A_{0} \wedge \square^{K} C_{0} \wedge \sigma^{K}\right)\right)$.

Proof. We repeat the proof of Lemma 3.8. Note that, by our reading conventions the antecedent $E_{1} \triangleright \neg\left(A_{0} \triangleright \neg C_{0}\right) \wedge \sigma$ should be read as $E_{1} \triangleright\left(\neg\left(A_{0} \triangleright \neg C_{0}\right) \wedge \sigma\right)$. We reason in $T$ and see that

$$
\begin{aligned}
\neg\left(A_{0} \triangleright \neg C_{0}\right) \wedge \sigma & \rightarrow \forall^{\text {Cut }} K \diamond\left(A_{0} \wedge \square^{K} C_{0}\right) \wedge \sigma \\
& \rightarrow \quad \forall^{\text {Cut }} K \diamond\left(A_{0} \wedge \square^{K} C_{0} \wedge \sigma^{K}\right) .
\end{aligned}
$$

As before, this final equation implies $\exists^{\text {Cut }} J \square\left(E_{1} \rightarrow \forall^{\text {Cut }} K \in J \diamond^{J}\left(A_{0} \wedge \square^{K} C_{0} \wedge\right.\right.$ $\left.\left.\sigma^{K}\right)\right)$.

With the following lemma we see that the $\mathcal{H}_{k+1}$ are an $\exists \Sigma_{1}^{b}$ encoding of information present in $E_{k+1} \triangleright \mathrm{Y}_{k}$ :

Lemma 3.13. Let $T$ be a theory containing $S_{2}^{1}$ and let the formulas $E_{i}, A_{i}$, and $C_{i}$ be arbitrary. For any number $k$ we have that

$$
T \vdash E_{k+1} \triangleright \mathrm{Y}_{k} \rightarrow \mathcal{H}_{k+1}
$$

Proof. By an external induction on $k$. For $k=0$ this is simply Lemma 3.8. For the inductive case we reason in $T$ and see that

$$
E_{k+2} \triangleright \mathrm{Y}_{k+1} \equiv E_{k+2} \triangleright \neg\left(A_{k+1} \triangleright \neg C_{k+1}\right) \wedge\left(E_{k+1} \triangleright \mathrm{Y}_{k}\right)
$$

By the inductive hypothesis we have that $E_{k+1} \triangleright \mathrm{Y}_{k} \rightarrow \mathcal{H}_{k+1}$ so that

$$
E_{k+2} \triangleright \mathrm{Y}_{k+1} \rightarrow E_{k+2} \triangleright \neg\left(A_{k+1} \triangleright \neg C_{k+1}\right) \wedge \mathcal{H}_{k+1}
$$

Since $\mathcal{H}_{k+1}$ is equivalent to an $\exists \Sigma_{1}^{b}$ formula, by Lemma 3.12 we see that

$$
E_{k+2} \triangleright \neg\left(A_{k+1} \triangleright \neg C_{k+1}\right) \wedge \mathcal{H}_{k+1} \rightarrow \mathcal{H}_{k+2}
$$

as was to be shown.

Moreover, the $\mathcal{H}_{k+1}$ formulas contain all the information to get the induction going as shown by the following lemma.

Lemma 3.14. Let $T$ be a theory containing $\mathrm{S}_{2}^{1}$ and let the formulas $E_{i}, A_{i}, B_{i}$, and $C_{i}$ be arbitrary. For any number $k$ we have that

$$
T \vdash\left(\mathrm{X}_{k}\right) \wedge \mathcal{H}_{k+1} \rightarrow E_{k+1} \triangleright \mathrm{Z}_{k}
$$


Proof. By induction on $k$ where the case $k=0$ is just lemma 3.10. For the inductive case, we reason in $T$ and assume $\left(\mathrm{X}_{k+1}\right) \wedge \mathcal{H}_{k+2}$.

From the definition of $\mathcal{H}_{k+2}$ we get

$$
\exists^{\text {Cut }} J_{k+2} \square\left(E_{k+2} \rightarrow \forall^{\text {Cut }} K_{k+2} \in J_{k+2} \diamond^{J_{k+2}}\left(A_{k+1} \wedge \square^{K_{k+2}} C_{k+1} \wedge \mathcal{H}_{k+1}^{K_{k+2}}\right)\right)
$$

so that $\exists^{\text {Cut }} J_{k+2} \forall^{\text {Cut }} K_{k+2} \square\left(E_{k+2} \rightarrow \diamond^{J_{k+2}}\left(A_{k+1} \wedge \square^{K_{k+2}} C_{k+1} \wedge \mathcal{H}_{k+1}^{K_{k+2}}\right)\right)$

whence

$$
\forall^{\text {Cut }} K_{k+2}\left(E_{k+2} \triangleright A_{k+1} \wedge \square^{K_{k+2}} C_{k+1} \wedge \mathcal{H}_{k+1}^{K_{k+2}}\right) .
$$

From $\mathrm{X}_{k+1}$ - which is by definition equal to $A_{k+1} \triangleright B_{k+1} \wedge\left(\mathrm{X}_{k}\right)$ - we find via Pudlák's lemma, our Lemma 2.2 a specific cut $\bar{K}_{k+2}$ such that for any formula $\sigma$ in $\Sigma_{1}$ we obtain $A_{k+1} \wedge \sigma^{\bar{K}_{k+2}} \triangleright B_{k+1} \wedge\left(\mathrm{X}_{k}\right) \wedge \sigma$. We can plug in this cut $\bar{K}_{k+2}$ to (19) to obtain via transitivity of $\triangleright$ that

$$
E_{k+2} \triangleright B_{k+1} \wedge\left(\mathrm{X}_{k}\right) \wedge \square C_{k+1} \wedge \mathcal{H}_{k+1}
$$

We are almost done but $B_{k+1} \wedge\left(\mathrm{X}_{k}\right) \wedge \square C_{k+1} \wedge \mathcal{H}_{k+1}$ is not quite equal to $\mathrm{Z}_{k+1}$ as was needed. The missing conjuncts are $E_{k+1} \triangleright A_{k}$ and $E_{k+1} \triangleright Z_{k}$. The first is easily seen to follow from $\mathcal{H}_{k+1}$ and the second follows from the inductive hypothesis applied to $\left(\mathrm{X}_{k}\right) \wedge \mathcal{H}_{k+1}$.

We are now ready to prove Theorem 3.7 that the whole hierarchy is arithmetically sound.

Theorem 3.15. Let $T$ be a theory containing $\mathrm{S}_{2}^{1}$ and let $A_{i}, B_{i}, C_{i}$ and $E_{i}$ be arbitrary arithmetical formulas. We have for each number $k$ that

$$
T \vdash \widetilde{\mathrm{R}}_{k} \text { id est } T \vdash \mathrm{X}_{k} \rightarrow \mathrm{Y}_{k} \triangleright \mathrm{Z}_{k} .
$$

Proof. By an external induction on $k$ where the base case is the soundness of $\widetilde{\mathrm{R}}_{0}$ which has been proven in [4. Thus, we reason in $T$ assuming $A_{k+1} \triangleright B_{k+1} \wedge\left(\mathrm{X}_{k}\right)$. We need to conclude that $Y_{k+1} \triangleright Z_{k+1}$. But $Y_{k+1}$ is nothing but $\neg\left(A_{k+1} \triangleright\right.$ $\left.\neg C_{k+1}\right) \wedge\left(E_{k+1} \triangleright \mathrm{Y}_{k}\right)$. By Lemma 3.13 we know that $\left(E_{k+1} \triangleright \mathrm{Y}_{k}\right) \rightarrow \mathcal{H}_{k+1}$. Using this and reasoning as before we obtain

$$
\begin{aligned}
\neg\left(A_{k+1} \triangleright \neg C_{k+1}\right) \wedge\left(E_{k+1} \triangleright \mathrm{Y}_{k}\right) & \rightarrow \forall^{\text {Cut }} K \diamond\left(A_{k+1} \wedge \square^{K} C_{k+1}\right) \wedge\left(E_{k+1} \triangleright \mathrm{Y}_{k}\right) \\
& \rightarrow \forall^{\text {Cut }} K \diamond\left(A_{k+1} \wedge \square^{K} C_{k+1}\right) \wedge \mathcal{H}_{k+1} \\
& \rightarrow \forall^{\text {Cut }} K \diamond\left(A_{k+1} \wedge \square^{K} C_{k+1} \wedge \mathcal{H}_{k+1}^{K}\right) .
\end{aligned}
$$

Consequently,

$$
\forall^{\text {Cut }} K\left(\neg\left(A_{k+1} \triangleright \neg C_{k+1}\right) \wedge\left(E_{k+1} \triangleright \mathrm{Y}_{k}\right) \triangleright A_{k+1} \wedge \square^{K} C_{k+1} \wedge \mathcal{H}_{k+1}^{K}\right) .
$$

This can be combined with Pudlák's Lemma on $A_{k+1} \triangleright B_{k+1} \wedge\left(\mathrm{X}_{k}\right)$ to obtain

$$
\neg\left(A_{k+1} \triangleright \neg C_{k+1}\right) \wedge\left(E_{k+1} \triangleright \mathrm{Y}_{k}\right) \triangleright B_{k+1} \wedge\left(\mathrm{X}_{k}\right) \wedge \square C_{k+1} \wedge \mathcal{H}_{k+1} .
$$

It is easy to see that $\mathcal{H}_{k+1}$ implies $E_{k+1} \triangleright A_{k}$. Moreover, Lemma 3.14 tells us that $\left(\mathrm{X}_{k}\right) \wedge \mathcal{H}_{k+1} \rightarrow E_{k+1} \triangleright \mathrm{Z}_{k}$ so that we may conclude

$$
\neg\left(A_{k+1} \triangleright \neg C_{k+1}\right) \wedge\left(E_{k+1} \triangleright \mathrm{Y}_{k}\right) \triangleright B_{k+1} \wedge\left(\mathrm{X}_{k}\right) \wedge \square C_{k+1} \wedge\left(E_{k+1} \triangleright A_{k}\right) \wedge\left(E_{k+1} \triangleright \mathrm{Z}_{k}\right)
$$

as was to be shown. 


\section{A broad series of principles}

In this section we present a different series of principles. We refer to this series as the broad series since the frame-conditions - see Figure 2- are typically represented over a broader area than the slim hierarchy as discussed above.

\subsection{A broad series}

In order to define the second series we first define a series of auxiliary formulas. For any $n \geq 1$ we define the schemata $\mathrm{U}_{n}$ as follows.

$$
\begin{aligned}
\mathrm{U}_{1} & :=\diamond \neg\left(D_{1} \triangleright \neg C\right), \\
\mathrm{U}_{n+2} & :=\diamond\left(\left(D_{n+1} \triangleright D_{n+2}\right) \wedge \mathrm{U}_{n+1}\right) .
\end{aligned}
$$

Now, for $n \geq 0$ we define the schemata $\mathrm{R}^{n}$ as follows.

$$
\begin{aligned}
\mathrm{R}^{0} & :=A \triangleright B \rightarrow \neg(A \triangleright \neg C) \triangleright B \wedge \square C, \\
\mathrm{R}^{n+1} & :=A \triangleright B \rightarrow\left(\mathrm{U}_{n+1} \wedge\left(D_{n+1} \triangleright A\right)\right) \triangleright B \wedge \square C .
\end{aligned}
$$

As an illustration we shall calculate the first four principles.

$$
\begin{aligned}
\mathrm{R}^{0} & :=A \triangleright B \rightarrow \neg(A \triangleright \neg C) \triangleright B \wedge \square C \\
\mathrm{R}^{1} & :=A \triangleright B \rightarrow \diamond \neg\left(D_{1} \triangleright \neg C\right) \wedge\left(D_{1} \triangleright A\right) \triangleright B \wedge \square C \\
\mathrm{R}^{2}:= & A \triangleright B \rightarrow \diamond\left[\left(D_{1} \triangleright D_{2}\right) \wedge \diamond \neg\left(D_{1} \triangleright \neg C\right)\right] \wedge\left(D_{2} \triangleright A\right) \triangleright B \wedge \square C \\
\mathrm{R}^{3}:= & A \triangleright B \rightarrow \diamond\left(\left(D_{2} \triangleright D_{3}\right) \wedge \diamond\left[\left(D_{1} \triangleright D_{2}\right) \wedge \diamond \neg\left(D_{1} \triangleright \neg C\right)\right]\right) \wedge\left(D_{3} \triangleright A\right) \\
& \quad B \wedge \square C
\end{aligned}
$$

While the series $\mathrm{R}_{i}$ did define a hierarchy in that $\mathrm{R}_{i+1} \vdash \mathrm{R}_{i}$, we shall see that no such relation holds for the series $\mathrm{R}^{i}$.

\subsection{Frame conditions}

It is not hard to determine the frame condition for the first couple of principles in this series and in Figure 2 we have depicted the first three frame-conditions. In this section we shall prove that the correspondence proceeds as expected. Informally, the frame condition for $\mathrm{R}^{n}$ shall be the universal closure of

$$
x_{n+1} R x_{n} \ldots R x_{0} R y_{0} S_{x_{1}} y_{1} \ldots S_{x_{n}} y_{n} S_{x_{n+1}} y_{n+1} R u \rightarrow y_{0} S_{x_{0}} u .
$$

In order to make this frame condition precise and prove it, we shall first recast it in a recursive fashion. In writing (20) recursively we shall use those variables that will emphasize the relation with (20). Of course, free variables can be renamed at the readers liking.

First, we start by introducing a relation $\mathcal{B}_{n}$ that captures the antecedent of (20). Note that this antecedent says that first there is a chain of points $x_{i}$ related by $R$, followed by a chain of points $y_{i}$ related by different $S$ relations. 

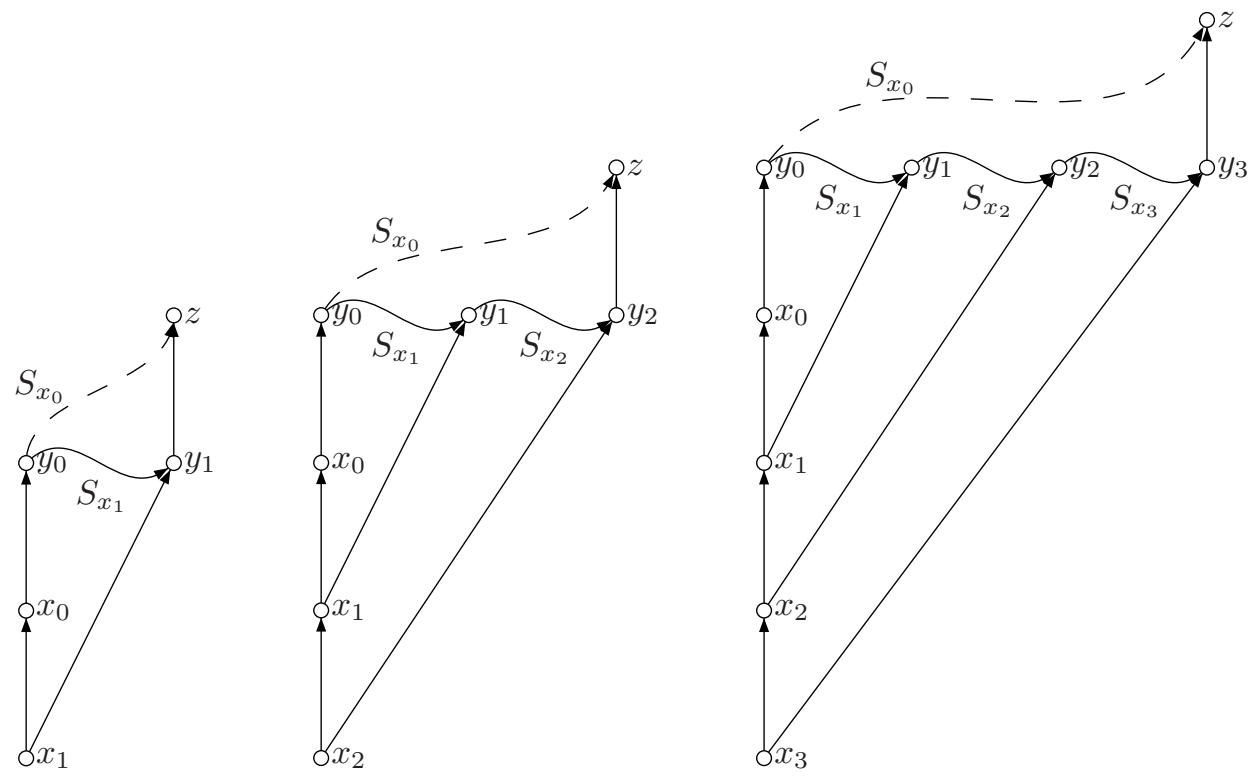

Figure 2: From left to right, this figure depicts the frame conditions $\mathcal{F}^{0}$ through $\mathcal{F}^{2}$ corresponding to $\mathrm{R}^{0}$ through $\mathrm{R}^{2}$. The reading convention is as always: if all the un-dashed relations are present as in the picture, then also the dashed relation should be there.

The relation $\mathcal{B}_{n}$ will be applied to the end-points of both chains where the condition on the intermediate points is imposed by recursion.

$$
\begin{aligned}
\mathcal{B}_{0}\left(x_{1}, x_{0}, y_{0}, y_{1}\right):=x_{1} R x_{1} R y_{0} S_{x_{1}} y_{1}, & \\
\mathcal{B}_{n+1}\left(x_{n+2}, x_{0}, y_{0}, y_{n+2}\right):= & \exists x_{n+1}, y_{n+1}\left(x_{n+2} R x_{n+1} \wedge \mathcal{B}_{n}\left(x_{n+1}, x_{0}, y_{0}, y_{n+1}\right)\right. \\
& \left.\wedge y_{n+1} S_{x_{n+2}} y_{n+2}\right) .
\end{aligned}
$$

For every $n \geq 0$ we can now define the first order frame condition $\mathcal{F}^{n}$ as follows.

$$
\mathcal{F}^{n}:=\forall x_{n+1}, x_{0}, y_{0}, y_{n+1}\left(\mathcal{B}_{n}\left(x_{n+1}, x_{0}, y_{0}, y_{n+1}\right) \Rightarrow \forall u\left(y_{n+1} R u \Rightarrow y_{0} S_{x_{0}} u\right)\right) .
$$

Sometimes we shall write $x_{n+1} \mathcal{B}_{n}\left[x_{0}, y_{0}\right] y_{n+1}$ conceiving the quaternary relation $\mathcal{B}_{n}$ as a binary relation indexed by the pair $x_{0}, y_{0}$. In what follows we let $F=\langle W, R, S\rangle$ be an arbitrary Veltman-frame. The next lemma follows from an easy induction on $n$.

Lemma 4.1. For each number $n$ we have that $\mathcal{B}_{n}\left[x_{0}, y_{0}\right] \subseteq R$, that is, if $x_{n+1} \mathcal{B}_{n}\left[x_{0}, y_{0}\right] y_{n+1}$, then $x_{n+1} R y_{n+1}$. 
To prove that $F \models \mathcal{F}^{n}$ implies $F \models \mathrm{R}^{n}$ we first need a technical lemma.

Lemma 4.2. Let $w \in W$ and $\Vdash$ be a forcing relation on $F$. If

$$
x_{k+1} \Vdash \mathrm{U}_{k+1} \wedge\left(D_{k+1} \triangleright A\right),
$$

then there exist $x_{0}, y_{0}$ and $y_{k+1}$ such that $\mathcal{B}_{k}\left(x_{k+1}, x_{0}, y_{0}, y_{k+1}\right), x_{0} R_{\Vdash}^{C} y_{0}$ and $y_{k+1} \Vdash A$.

Proof. Induction on $k$. If $k=0$ then $\mathrm{U}_{k+1}=\diamond \neg\left(D_{1} \triangleright \neg C\right)$ and the statement is easily checked. For the inductive case, we assume

$$
x_{k+2} \Vdash \mathrm{U}_{k+2} \wedge\left(D_{k+2} \triangleright A\right) .
$$

Recall that $\mathrm{U}_{k+2}:=\diamond\left(\left(D_{k+1} \triangleright D_{k+2}\right) \wedge \mathrm{U}_{k+1}\right)$. Thus, there exists some $x_{k+1}$ with $x_{k+2} R x_{k+1}$ and

$$
x_{k+1} \Vdash\left(D_{k+1} \triangleright D_{k+2}\right) \wedge \mathrm{U}_{k+1} .
$$

Applying the (IH) (with $D_{k+2}$ substituted for $A$ ) we find $x_{0}, y_{0}$ and $y_{k+1}$ with $\mathcal{B}_{k}\left(x_{k+1}, x_{0}, y_{0}, y_{k+1}\right), x_{0} R_{\Vdash}^{C} y_{0}$ and $y_{k+1} \Vdash D_{k+2}$. As $\mathcal{B}_{k}\left(x_{k+1}, x_{0}, y_{0}, y_{k+1}\right)$ we get $x_{k+1} R y_{k+1}$ (Lemma 4.1). Since we had $x_{k+2} R x_{k+1}$ we see that $x_{k+2} R y_{k+1} \Vdash$ $D_{k+2}$, and since $x_{k+2} \Vdash D_{k+2} \triangleright A$, we find some $y_{k+2}$ with $y_{k+1} S_{x_{k+2}} y_{k+2}$ and $y_{k+2} \Vdash A$. By definition of $\mathcal{B}_{k+1}$ we have $\mathcal{B}_{k+1}\left(x_{k+2}, x_{0}, y_{0}, y_{k+2}\right)$.

Corollary 4.3. If $F \models \mathcal{F}^{n}$ then $F \models \mathrm{R}^{n}$.

Proof. Induction on $n$. For $n=0$ this is known (see [4), so we assume $n>$ 0 . Let $\Vdash$ be a forcing relation, let $x_{n+1}, x_{n} \in W$ and assume $x_{n+1} \Vdash A \triangleright$ $B, x_{n+1} R x_{n}$ and $x_{n} \Vdash \mathrm{U}_{n} \wedge\left(D_{n} \triangleright A\right)$. By Lemma 4.2 we find $x_{0}, y_{0}$ and $y_{n}$ such that $\mathcal{B}_{n-1}\left(x_{n}, x_{0}, y_{0}, y_{n}\right)$, with $x_{0} R_{\Vdash}^{C} y_{0}$ and $y_{n} \Vdash A$. We have that $\mathcal{B}_{n-1}\left(x_{n}, x_{0}, y_{0}, y_{n}\right)$ implies $x_{n} R y_{n}$ (Lemma 4.1) and thus since $x_{n+1} R x_{n}$ we also we have $x_{n+1} R y_{n} \Vdash A$. By assumption $x_{n+1} \vdash A \triangleright B$ so that for some $y_{n+1}$ we have $y_{n} S_{x_{n+1}} y_{n+1} \Vdash B$. Clearly, we also have $x_{n} S_{x_{n+1}} y_{n+1}$ so that we are done if we have shown that $y_{n+1} \Vdash \square C$. To this purpose, we choose some $u$ with $y_{n+1} R u$. Since we have that $\mathcal{B}_{n}\left(x_{n+1}, x_{0}, y_{0}, y_{n+1}\right)$, by $\mathcal{F}^{n}$ we have also $y_{0} S_{x_{0}} u$. But $x_{0} R_{\Vdash}^{C} y_{0}$ and thus we have $u \Vdash C$, as required.

To prove the converse implication, we start again with a technical lemma. As before we shall denote by $\boldsymbol{a}, \boldsymbol{b}, \boldsymbol{c}$, and $\boldsymbol{d}_{k}$, propositional variables that shall play the role of the $A, B, C$ and $D_{k}$ respectively in the principles $\mathrm{R}^{n}$. Let $\overline{\mathrm{U}}_{k}$ denote the formula that arises by simultaneously substituting $\boldsymbol{c}$ for $C$ and $\boldsymbol{d}_{k}$ for $D_{k}$ in $\mathrm{U}_{k}$.

Lemma 4.4. Let $\left\{\boldsymbol{a}, \boldsymbol{c}, \boldsymbol{d}_{1}, \ldots, \boldsymbol{d}_{k+1}\right\}$ be a collection of distinct propositional variables. If $F \models \mathcal{B}_{k}\left(x_{k+1}, x_{0}, y_{0}, y_{k+1}\right)$, then there exists a forcing relation $\Vdash$ on $F$ such that

$$
\text { 1. } x_{k+1} \Vdash \overline{\mathrm{U}}_{k+1} \wedge\left(\boldsymbol{d}_{k+1} \triangleright \boldsymbol{a}\right) \text {; }
$$




$$
\begin{aligned}
& \text { 2. } x \Vdash \boldsymbol{c} \text { iff } y_{0} S_{x_{0}} x ; \\
& \text { 3. } x \Vdash \boldsymbol{a} \Leftrightarrow x=y_{k+1} \text {; } \\
& \text { 4. } x \nVdash \boldsymbol{p} \text { for any } \boldsymbol{p} \notin\left\{\boldsymbol{d}_{1}, \ldots, \boldsymbol{d}_{k+1}, \boldsymbol{c}, \boldsymbol{a}\right\} \text {. }
\end{aligned}
$$

Proof. The idea is very simple using the informal description of $\mathcal{B}_{k}$ being the antecedent of (20). We define a valuation $\Vdash$ so that $\boldsymbol{d}_{i+1}$ is only true at $y_{i}$ and $\boldsymbol{a}$ is only true at $y_{k+1}$. Moreover, we define $x \Vdash \boldsymbol{c}$ iff $y_{0} S_{x_{0}} x$ and $x \nVdash \boldsymbol{p}$ for any $\boldsymbol{p} \notin\left\{\boldsymbol{d}_{1}, \ldots, \boldsymbol{d}_{k+1}, \boldsymbol{c}, \boldsymbol{a}\right\}$. It is not hard to see that $x_{k+1} \Vdash \overline{\mathrm{U}}_{k+1} \wedge\left(\boldsymbol{d}_{k+1} \triangleright \boldsymbol{a}\right)$ for this valuation $\Vdash$.

To make the argument precise, we proceed by induction on $k$. If $k=0$ then $\mathcal{B}_{k}\left(x_{1}, x_{0}, y_{0}, y_{1}\right)$ simply means $x_{1} R x_{0} R y_{0} S_{x_{1}} y_{1}$ and we define

$$
x \Vdash \boldsymbol{a} \Leftrightarrow x=y_{1}, \quad x \Vdash \boldsymbol{c} \Leftrightarrow y_{0} S_{x_{0}} x \quad \text { and }, \quad x \Vdash \boldsymbol{d}_{1} \Leftrightarrow x=y_{0} .
$$

The lemma is easily checked if we further define $x \nVdash \boldsymbol{p}$ for any $\boldsymbol{p} \notin\left\{\boldsymbol{d}_{1}, \boldsymbol{c}, \boldsymbol{a}\right\}$.

For the inductive case we consider $k>0$. Then $\mathcal{B}_{k}\left(x_{k+1}, x_{0}, y_{0}, y_{k+1}\right)$ implies that there are $x_{k}$ and $y_{k}$ such that

$$
x_{k+1} R x_{k} \mathcal{B}_{k-1}\left[x_{0}, y_{0}\right] y_{k} S_{x_{k+1}} y_{k+1} .
$$

The (IH) (with $\boldsymbol{d}_{k+1}$ substituted for $\boldsymbol{a}$ ) gives a forcing relation $\Vdash$ such that

$$
x_{k} \Vdash \overline{\mathrm{U}}_{k} \wedge\left(\boldsymbol{d}_{k} \triangleright \boldsymbol{d}_{k+1}\right), \quad x_{0} R_{\Vdash}^{\boldsymbol{c}} y_{0}, \quad x \Vdash \boldsymbol{d}_{k+1} \Leftrightarrow x=y_{k}
$$

and $x \nVdash \boldsymbol{p}$ for $\boldsymbol{p} \notin\left\{\boldsymbol{d}_{1}, \ldots, \boldsymbol{d}_{k+1}, \boldsymbol{c}\right\}$. So we have $x_{k+1} \Vdash \diamond\left(\overline{\mathrm{U}}_{k} \wedge\left(\boldsymbol{d}_{k} \triangleright \boldsymbol{d}_{k+1}\right)\right)$; in other words $x_{k+1} \Vdash \overline{\mathrm{U}}_{k+1}$. We now define $\Vdash^{\prime}$ as follows

$$
x \Vdash^{\prime} \boldsymbol{a} \Leftrightarrow x=y_{k+1} \quad \text { and } \quad x \Vdash^{\prime} \boldsymbol{p} \Leftrightarrow x \Vdash \boldsymbol{p} \text { for } \boldsymbol{p} \neq \boldsymbol{a} .
$$

Clearly, the properties $x_{k} \Vdash \overline{\mathrm{U}}_{k} \wedge\left(\boldsymbol{d}_{k} \triangleright \boldsymbol{d}_{k+1}\right), a R_{\Vdash}^{\boldsymbol{c}} b, x \Vdash \boldsymbol{d}_{k+1} \Leftrightarrow x=y_{k}$ simply extend to $\Vdash^{\prime}$ and likewise we have that $x \nVdash^{\prime} \boldsymbol{p}$ for any $\boldsymbol{p} \notin\left\{\boldsymbol{d}_{1}, \ldots, \boldsymbol{d}_{k+1}, \boldsymbol{c}, \boldsymbol{a}\right\}$. Moreover, we now have $x_{k+1} \Vdash^{\prime} \boldsymbol{d}_{k+1} \triangleright \boldsymbol{a}$ as well.

As a corollary to this lemma, we can now obtain the full the frame conditions for the principles $\mathrm{R}^{n}$.

Theorem 4.5. For each number $n$ we have $F \models \mathcal{F}^{n}$ iff $F \models \mathrm{R}^{n}$.

Proof. The $\Rightarrow$ direction is just Corollary 4.3 so we focus on the other direction. Thus, we suppose that $F \models \mathrm{R}^{n}$, consider any $x_{n+1}, x_{0}, y_{0}, y_{n+1} \in W$ with $\mathcal{B}_{n}\left(x_{n+1}, x_{0}, y_{0}, y_{n+1}\right)$ and set out to show that for any $u$ with $y_{n+1} R u$ we have $y_{0} S_{x_{0}} u$. We now apply Lemma 4.4 and simultaneously substitute $\boldsymbol{a}$ for $\boldsymbol{d}_{n+1}$ and $\boldsymbol{b}$ for $\boldsymbol{a}$ to see that there exists a forcing relation $\Vdash$ such that

$$
x_{n+1} \Vdash \overline{\mathrm{U}}_{n+1}\left[\boldsymbol{d}_{n+1} / \boldsymbol{a}\right] \wedge(\boldsymbol{a} \triangleright \boldsymbol{b}), \quad x \Vdash \boldsymbol{c} \Leftrightarrow y_{0} S_{x_{0}} x \quad \text { and } \quad x \Vdash \boldsymbol{b} \Leftrightarrow x=y_{n+1} .
$$

Since $n=0$ is known, we assume $n>0$. Thus, we find $x_{n}$ with $x_{n+1} R x_{n}$ and $x_{n} \Vdash \overline{\mathrm{U}}_{n-1} \wedge \boldsymbol{d}_{n} \triangleright \boldsymbol{a}$ (note that $\overline{\mathrm{U}}_{n-1}\left[\boldsymbol{d}_{n+1} / \boldsymbol{a}\right]=\overline{\mathrm{U}}_{n-1}$ ). Using $F \models \overline{\mathrm{R}}^{n}$ we see that there must exist some $x$ with $x \Vdash \boldsymbol{b} \wedge \square \boldsymbol{c}$. But $y_{n+1}$ is the only world that forces $\boldsymbol{b}$ thus necessarily $y_{n+1} \Vdash \square \boldsymbol{c}$. By the choice of $\Vdash$ we thus have that if $y_{n+1} R u$ then $y_{0} S_{x_{0}} u$. 
Using the frame condition we readily see that the broad series of principles does not define a hierarchy.

Corollary 4.6. For $n \neq m$ we have $\mathrm{ILR}^{n} \nvdash \mathrm{ILR}^{m}$.

Proof. For each $m \neq n$ it is easy to exhibit a frame $F$ so that $F \models \mathcal{F}^{n}$ but $F \not \models \mathcal{F}^{m}$.

\subsection{Arithmetical soundness}

We will now see that all the principles $\mathrm{R}^{n}$ are arithmetically sound and begin with a simple lemma.

Lemma 4.7. For any theory $T$ extending $\mathrm{S}_{2}^{1}$ and any natural number $n>0$, we have that

$$
T \vdash \mathrm{U}_{n} \rightarrow \forall{ }^{\text {Cut }} K \diamond\left(D_{n} \wedge \square^{K} C\right) .
$$

Proof. We proceed by induction on $n$ and first consider $n=1$. Thus, we reason in $T$ and assume $\mathrm{U}_{1}$, that is, $\diamond \neg\left(D_{1} \triangleright \neg C\right)$. We conclude by Lemma 2.6 that $\diamond \forall^{\text {Cut }} K \diamond\left(D_{1} \wedge \square^{K} C\right)$, whence $\forall{ }^{\text {Cut }} K \diamond \diamond\left(D_{1} \wedge \square^{K} C\right)$ and also

$$
\forall^{\text {Cut }} K \diamond\left(D_{1} \wedge \square^{K} C\right)
$$

as was to be shown.

Next, we consider the inductive case, again reasoning in $T$ and assuming $\mathrm{U}_{n+1}$ which is $\diamond\left(\left(D_{n} \triangleright D_{n+1}\right) \wedge \mathrm{U}_{n}\right)$. Reasoning inside the $\diamond$, by the (IH) we conclude from $\mathrm{U}_{n}$ that

$$
\forall^{\text {Cut }} J \diamond\left(D_{n} \wedge \square^{J} C\right) \text {. }
$$

By Lemma 2.8 we obtain from $D_{n} \triangleright D_{n+1}$ that

$$
\forall^{\text {Cut }} K \exists^{\text {Cut }} J D_{n} \wedge \square^{J} C \triangleright D_{n+1} \wedge \square^{K} C .
$$

Combining

$$
D_{n} \wedge \square^{J} C \triangleright D_{n+1} \wedge \square^{K} C \rightarrow\left(\diamond\left(D_{n} \wedge \square^{J} C\right) \rightarrow \diamond\left(D_{n+1} \wedge \square^{K} C\right)\right)
$$

with (21) and (22) under a $\diamond$ we conclude that

$$
\begin{aligned}
\diamond\left(\left(D_{n} \triangleright D_{n+1}\right) \wedge \mathrm{U}_{n}\right) & \rightarrow \diamond\left(\forall^{\text {Cut }} K \diamond\left(D_{n+1} \wedge \square^{K} C\right)\right) \\
& \rightarrow \forall^{\text {Cut }} K \diamond\left(\diamond\left(D_{n+1} \wedge \square^{K} C\right)\right) \\
& \rightarrow \quad \forall^{\text {Cut }} K \diamond\left(D_{n+1} \wedge \square^{K} C\right)
\end{aligned}
$$

as was to be shown.

With this lemma, we can now prove the soundness of the series $\mathrm{R}^{n}$.

Theorem 4.8. For each natural number $n$ we have that $\mathrm{R}^{n}$ is arithmetically sound in any theory $T$ extending $\mathrm{S}_{2}^{1}$. 
Proof. Since we already know that $\mathrm{R}^{0}$ is sound, we consider $n>0$. We reason in $T$, assume $A \triangleright B$ and set out to prove $\mathrm{U}_{n} \wedge\left(D_{n} \triangleright A\right) \triangleright B \wedge \square C$. By Pudlák's Lemma we get

$$
\exists^{\text {Cut }} J A \wedge \square^{J} C \triangleright B \wedge \square C .
$$

On the other hand, by the generalization of Pudlák's Lemma (Lemma 2.8) applied to $D_{n} \triangleright A$ we obtain that $\forall^{\text {Cut }} J \exists^{\text {Cut }} K D_{n} \wedge \square^{K} C \triangleright A \wedge \square^{J} C$ so that

$$
\forall^{\text {Cut }} J \exists^{\text {Cut }} K\left(\diamond\left(D_{n} \wedge \square^{K} C\right) \rightarrow \diamond\left(A \wedge \square^{J} C\right)\right) .
$$

By Lemma 4.7 we see that $\mathrm{U}_{n} \rightarrow \forall{ }^{\text {Cut }} K \diamond\left(A \wedge \square^{K} C\right)$. Combining these last two observations, we see that

$$
\mathrm{U}_{n} \wedge\left(D_{n} \triangleright A\right) \rightarrow \forall^{\mathrm{Cut}} J \diamond\left(A \wedge \square^{J} C\right)
$$

so that

$$
\forall^{\text {Cut }} J \mathrm{U}_{n} \wedge\left(D_{n} \triangleright A\right) \triangleright A \wedge \square^{J} C .
$$

Combining this with (23) yields $\mathrm{U}_{n} \wedge\left(D_{n} \triangleright A\right) \triangleright B \wedge \square C$ as was to be shown.

\section{On the core interpretability logic IL(All)}

Apart from the principles mentioned earlier in this paper the literature has considered various other principles too. Some of those are

$$
\begin{aligned}
\mathrm{W}: & A \triangleright B \rightarrow A \triangleright B \wedge \square \neg A \\
\mathrm{~W}^{*}: & A \triangleright B \rightarrow B \wedge \square C \triangleright B \wedge \square C \wedge \square \neg A \\
\mathrm{P} & : A \triangleright \vee B \rightarrow \square(A \triangleright B) \\
\mathrm{R}: & A \triangleright B \rightarrow \neg(A \triangleright \neg C) \triangleright B \wedge \square C
\end{aligned}
$$

In [13, IL(All) was conjectured to be ILW. In [15] this conjecture was falsified and strengthened to a new conjecture, namely that ILW*, which is a proper extension of $\mathbf{I L W}$, is $\mathbf{I L}(\mathrm{All})$. In $[8]$ it was proven that the logic $\mathbf{I L W}^{*} \mathrm{P}_{0}$ is a proper extension of $\mathbf{I L W}$, and that $\mathbf{I L W} \mathbf{P}_{0}$ is a subsystem of $\mathbf{I L}(\mathrm{All})$ (we write $\mathbf{I L W}^{*} \mathrm{P}_{0}$ instead of $\left.\mathbf{I L}\left\{\mathbf{W}^{*}, \mathrm{P}_{0}\right\}\right)$. This falsified the conjecture from [15]. In [8] it is also conjectured that $\mathbf{I L W} \mathbf{P}_{0}$ is not the same as $\mathbf{I L}(\mathrm{All})$.

In [7] it is conjectured that $\mathbf{I L W} \mathbf{P}_{0}=\mathbf{I L}($ All $)$ and this conjecture was refuted in 4 by proving that the logic ILRW is a subsystem of $\mathbf{I L ( A l l )}$ and a proper extension of $\mathbf{I L W} \mathbf{P}_{0}$.

It is easy to see that $A \triangleright \diamond B \rightarrow \square(A \triangleright \diamond B) \in$ ILP $\cap$ ILM. In [16] it was shown however that $A \triangleright \diamond B \rightarrow \square(A \triangleright \diamond B) \notin \mathbf{I L}$ (All) thereby lowering the upper bound $\mathbf{I L}($ All $) \subseteq \mathbf{I L P} \cap \mathbf{I L M}$. Since $A \triangleright \diamond B \rightarrow \square(A \triangleright \diamond B)$ is reminiscent of the modally incomplete principle $\mathrm{P}_{0}$, we remark here that the principle

$$
A \triangleright B \rightarrow \neg(A \triangleright \triangleright C) \triangleright B \wedge \square \neg C
$$


implies $A \triangleright \diamond B \rightarrow \square(A \triangleright \diamond B)$ so that it cannot be in $\mathbf{I L}($ All $)$ either.

The current paper raises the previously known lower bound of IL(All). However, it seems unlikely that this will be the end of the story and the two series presented here seem amenable for interactions. Just by mere inspection of the frame conditions we observe that

$$
\begin{aligned}
& \mathcal{F}_{n}=\forall w, x, y, z\left(\mathcal{B}_{0}(w, x, y, z) \Rightarrow \mathcal{G}_{n}(x, y, z)\right), \\
& \mathcal{F}^{n}=\forall w, x, y, z\left(\mathcal{B}_{n}(w, x, y, z) \Rightarrow \mathcal{G}_{0}(x, y, z)\right) .
\end{aligned}
$$

suggesting possible interactions. For example, a combination of $R^{1}$ and $R_{1}$ could yield

$$
A \triangleright B \rightarrow(C \triangleright A) \wedge \diamond \neg(C \triangleright \neg D) \wedge(E \triangleright \diamond F) \triangleright B \wedge \square D \wedge(E \triangleright F) .
$$

We note that the two series presented in this paper only spoke of $S$ relations that were imposed by the frame conditions. This suggests that a new conjecture can be formulated.

In words the conjecture is expressed as follows. First we single out the second order frame conditions that are inherent to provability and interpretability. These are the converse well-foundedness of the $R$ relation as expressed by Löb's axiom $\square(\square A \rightarrow A) \rightarrow \square A$ and the converse well-foundedness of $R \circ S_{x}$ as expressed by W. Over these frames, we will further impose the existence of all $S_{x}$ relations that are forced to be there in virtue of both the ILP and the ILM frame condition. The logic of those frames is put forward as the new conjecture for IL(All).

To make this conjecture mathematically precise, we will introduce some notation. Let $\mathfrak{F}$ be a class of $\mathbf{I L}$-frames. By $\mathbf{I L}[\mathfrak{F}]$ we shall denote the interpretability logic corresponding to this class. That is,

$$
\mathbf{I L}[\mathfrak{F}]:=\left\{A \mid \forall F \in \mathfrak{F} \forall^{\text {valuation }} V\langle F, V\rangle \models A\right\} .
$$

Let $F(x, y, z)$ denote any sentence - first or higher order - in the language with a binary relation $R$ and infinitely many indexed binary relations $S_{u}$. We now define the following class of conditions

$$
\begin{aligned}
& \mathcal{C}_{\mathrm{ILP}_{S} \mathrm{ILM}}:=\quad\left\{F(x, y, z) \rightarrow x S_{y} z \mid \mathrm{ILP} \models F(x, y, z) \rightarrow x S_{y} z \wedge\right. \\
& \text { ILM } \left.\models F(x, y, z) \rightarrow x S_{y} z\right\} .
\end{aligned}
$$

We wrote ILP $\models F(x, y, z) \rightarrow y S_{x} z$ to denote that for any Veltman frame $\mathcal{F}$ for which $\mathcal{F} \models$ ILP we also have $\mathcal{F} \models F(x, y, z) \rightarrow y S_{x} z$. Likewise, we speak of ILM $\models F(x, y, z) \rightarrow y S_{x} z$. Of course, in this context the condition $F(x, y, z) \rightarrow x S_{y} z$ is equivalent to its universal closure. The class $\mathcal{C}_{\mathbf{I L P} \cap \cap_{S} \mathbf{I L M}}$ should thus capture all the $S_{x}$ relations that are imposed both because of ILM and of ILP frame conditions. We now define

$$
\mathfrak{A} \mathfrak{H I}:=\left\{\mathcal{F} \models \mathbf{I L W} \mid \forall C \in \mathcal{C}_{\mathbf{I L P}_{S}{ }_{S} \mathbf{I L M}} F \models C\right\}
$$

The second author poses the new conjecture 
Conjecture 5.1. IL $(\mathrm{All})=\mathrm{IL}[\mathfrak{A} \mathfrak{l l}]$.

It is easy to formulate the conjecture where the antecedent $F(x, y, z)$ is replaced by a set of sentences rather than a single sentence yet it seems hard to imagine that this is needed. Note that the conjecture only speaks of principles related to imposed $S$ relations. For example, this will leave out a principle like $A \triangleright B \rightarrow(\diamond A \wedge \square \square C \triangleright B \wedge \square C)$ as formulated in [7] and whose frame condition is $x R y R z S_{x} u R v \rightarrow \exists w y R w R v$.

As the referee did, we remark that with the current paper out, it seems unlikely that $\mathbf{I L}$ (All) will have a nice axiomatization. It may occur however that resorting to a richer language may significantly simplify the answer. In 9 the second author and Visser looked at such a richer language where constants for particular definable cuts were available. In the light of the current paper, we expect that studies as in [9] will gain increasing importance.

\section{Acknowledgement}

The authors are grateful to Albert Visser, Lev Beklemishev and Volodya Shavrukov for their support, encouragement, discussion, questions and improvements. Further, we would like to thank the anonymous referee for his/her meticulous, informative and constructive report pointing out many inaccuracies and suggestions for improvements. Incorporating the comments and suggestions has much improved the submitted paper.

A pleasant feature of the referee report has been its constructive spirit: in many cases where a problem was spotted, the referee has been so kind and diligent to also suggest a possible solution. Furthermore, the referee takes no blame in the delay between communication and acceptance of the paper which is entirely the fault of the authors: it got forgotten about once and later it got buried under more urgent but less interesting tasks.

\section{References}

[1] A. Berarducci. The interpretability logic of peano arithmetic. Journal of Symbolic Logic, 55(3):1059-1089, 1990.

[2] S. Buss. Bounded arithmetic. Bibliopolis, Napoli, 1986.

[3] D.H.J. de Jongh and F. Veltman. Provability logics for relative interpretability. In P.P. Petkov, editor, Mathematical Logic, Proceedings of the Heyting 1988 summer school in Varna, Bulgaria, pages 31-42. Plenum Press, Boston, New York, 1990.

[4] E. Goris and J. J. Joosten. A new principle in the interpretability logic of all reasonable arithmetical theories. Logic Journal of the IGPL, 19(1):14-17, 2011.

[5] P. Hájek and P. Pudlák. Metamathematics of First Order Arithmetic. Springer-Verlag, Berlin, Heidelberg, New York, 1993. 
[6] J. J. Joosten. On formalizations of the Orey-Hájek characterization for interpretability. In P. Cegielski, A. Enayat, and R. Kossak, editors, Studies in Weak Arithmetics, pages 57-90. CSLI Publications, Stanford, 2016.

[7] J. J. Joosten and A. Visser. The interpretability logic of all reasonable arithmetical theories. The new conjecture. Erkenntnis, 53(1-2):3-26, 2000.

[8] J.J. Joosten. Towards the interpretability logic of all reasonable arithmetical theories. Master's thesis, University of Amsterdam, 1998.

[9] J.J. Joosten and A. Visser. How to derive principles of interpretability logic, A toolkit. In J. van Benthem, F. Troelstra, A. Veltman, and A. Visser, editors, Liber Amicorum for Dick de Jongh. Intitute for Logic, Language and Computation, 2004. Electronically published, ISBN: 9057761289.

[10] J. Mycielski, P. Pudlák, and A.S. Stern. A lattice of chapters of mathematics (interpretations between theorems), volume 426 of Memoirs of the American Mathematical Society. AMS, Providence, Rhode Island, 1990.

[11] V. Y. Shavrukov. The logic of relative interpretability over Peano arithmetic. Preprint, Steklov Mathematical Institute, Moscow, 1988. In Russian.

[12] A. Tarski, A. Mostowski, and R. Robinson. Undecidable Theories. NorthHolland, Amsterdam, 1953.

[13] A. Visser. Preliminary notes on interpretability logic. Technical Report LGPS 29, Department of Philosophy, Utrecht University, 1988.

[14] A. Visser. Interpretability logic. In P.P. Petkov, editor, Mathematical Logic, Proceedings of the Heyting 1988 summer school in Varna, Bulgaria, pages 175-209. Plenum Press, Boston, New York, 1990.

[15] A. Visser. The formalization of interpretability. Studia Logica, 50(1):81106, 1991.

[16] A. Visser. An overview of interpretability logic. In M. Kracht, M. de Rijke, and H. Wansing, editors, Advances in modal logic '96, pages 307-359. CSLI Publications, Stanford, CA, 1997.

[17] A. Visser. The arithmetics of a theory. Notre Dame J. Formal Logic, 56(1):81-119, 2015. 